\title{
Determination of reservoir properties through the use of computed X-ray microtomography - eolian sandstone examples
}

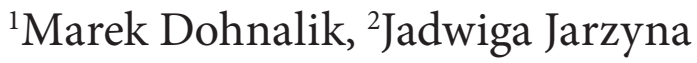 \\ ${ }^{1}$ Oil and Gas Institute - National Research Institute; ul. Lubicz 25a, 31-503 Krakow, Poland; e-mail: dohnalik@inig.pl \\ ${ }^{2}$ AGH University of Science and Technology, Faculty of Geology, Geophysics and Environment Protection, Department \\ of Geophysics; al. A. Mickiewicza 30,30-059 Krakow, Poland; e-mail: jarzyna@agh.edu.pl
}

(c) 2015 Authors. This is an open access publication, which can be used, distributed and reproduced in any medium according to the Creative Commons CC-BY 4.0 License requiring that the original work has been properly cited.

Received: 18 April 2014; accepted: 11 August 2015

\begin{abstract}
The possibility of examining rock pore structure by the means of computed X-ray microtomography was presented. Parameters characterizing the pore structure, such as: porosity and coefficient of homogeneity of pore structure by local porosity examination, box counting dimension, mean chord length, normalized Euler number, as well as coordination number were all determined through pore structure image analyses. Complementary methods, such as helium pycnometry, mercury injection capillary pressure and NMR were used to determine the porosity along with other factors to make the comparison and determine mutual relationships between petrophysical properties obtained from various sources.

The study covered the Rotliegend sandstones of eolian origin. Laboratory investigation of the reservoir properties was focused on three areas, according to their geological regions, where the Rotliegend sediments showed changeability in geological features and reservoir properties. Computed X-ray microtomography showed differentiation between the investigated areas, with respect to their pore structure and porosity development. This differentiation was confirmed by means of other applied laboratory methods.
\end{abstract}

Keywords: computed X-ray microtomography reservoir rocks, petrophysical properties, Rotliegend sandstones

\section{INTRODUCTION}

Hydrocarbon deposits, which are easily accessible or occur in rocks with good reservoir properties, have already been discovered in Poland and many countries around the world. As such, prospecting in recent years prospecting has focused on unconventional sources, where gas and oil are trapped within rock formations of poor reservoir properties, i.e. porosity less than $10 \%$ and permeability less than $0.1 \mathrm{mD}$ (Law \& Spencer 1993). New conditions of hydrocarbon exploitation necessitate the development of laboratory studies in order to be able to better recognize rock properties.

Prospecting for hydrocarbons and water, under increasingly difficult geological conditions, needs improvement in research techniques and refining pore space description methods. The key factor for the prediction of reservoir properties from laboratory studies is their very detailed description of the pore space structure of rocks. In petro-physics, within the space of a few dozen years, the employment of new technologies, applied successfully applied in other disciplines, has been observed. Two of the methods which were used in this study were 
originally applied in medicine and chemistry (micro-CT and NMR).

The goal of this paper is to present the possibility of examining the pore structure of sandstones by means of the X-ray computed microtomography (micro-CT) method. Parameters characterizing the pore structure, i.e. porosity and coefficient of homogeneity of pore structure by local porosity examination, box counting dimension, mean chord length, normalized Euler number, and coordination number were all determined from micro-CT measurements, through pore structure image analyses. The device used in the research gave images of the inner structure of the rocks, with the resolution of $5.8 \mu \mathrm{m}$. However, pore diameters of the investigated rocks ranged from a few $\mathrm{nm}$ to a few $\mathrm{mm}$. Hence, part of the pore structure (in some cases even a major part) could have been omitted in microtomography imaging and further analysis. To verify the micro-CT results, some of the parameters were compared to those calculated with the use of proven methods, including helium porosimetry, mercury injection capillary pressure and nuclear magnetic resonance.

In the referenced literature, there are examples illustrating the application of micro-CT techniques, in order to show inner structure of various materials (Osher \& Schladitz 2009, Golab et al. 2013, Nadeev et al. 2013), among them rocks. In this paper, the Rotliegend rocks were selected to be examined using this new technique to improve reservoir properties determination. Numerous gas deposits were discovered in the Rotliegend clastic formation in Poland and in neighbouring countries. Hence, this formation is still of interest as a perspective reservoir (Buniak et al. 2008a, 2008b).

The Permian (the Rotliegend and Zechstein) basin on Poland's territory constituted the eastern part of the south Permian basin, extending over Western and Central Europe (Karnkowski 1999). The Rotliegend continental basin was essentially the result of extensive tectonic regime and geology of the sub-Permian basement (Pokorski 1997). The environment of deposition for the Rotliegend sediments was a desert (Buniak \& Mikołajewski 1997, Pokorski 1997, Kiersnowski 1998, Aksamitowska 2003).

The biggest region of the Upper Rotliegend basin in Poland is the Central Basin (Pokorski 1997), which extends over the present Mid-Polish
Anticlinorium, Marginal Synclinorium and Szczecin-Łódź-Miechów Synclinorium, as well as the northern part of the Fore-Sudetic Monocline (Fig. 1). A dune area, called an eastern erg, was a characteristic element of the southern part of the Central Basin. In this area, sediments of the eolian depositional system dominate (Kiersnowski 1997). The Wolsztyn High was a natural barrier for dune fields. The development of eolian forms was probably limited by alluvial fans, which are considered to have occurred in the marginal zone (Kwolek et al. 2004). Eolian deposits terminated the Rotliegend deposition over the whole area. At the end of sedimentation, just before transgression of the Zechstein Sea, the dune field was at its maximum extent.

Terrigenous deposits occurred in the basin in the form of claystones, mudstones, sandstones, and conglomerates. Clastic deposits represented three main depositional systems, related to desert sedimentation: the fluvial depositional system (alluvial fan facies, channel fluvial facies), eolian depositional system (dune facies, interdune facies) and the lacustral depositional system (clay playa facies, sand playa facies, marginal playa facies) (Kiersnowski 1997). The study covered the Rotliegend sandstones of the eolian origin

Data used in this study were acquired from boreholes drilled in the area. Core samples were taken at intervals, and laboratory measurements were made. The comprehensive interpretation of logs from the wells provided volumes of minerals composing rocks, porosity, permeability and gas saturation.

\section{CHARACTERISTICS OF THE STUDY MATERIAL}

The sandstones studied were collected from two selected areas: one located in the marginal part of the eastern erg (Czarna Wieś - Parzęczewo), and the other located in its middle part (Siekierki Kromolice - Miłosław). The Rotliegend rocks of the Czarna Wieś - Parzęczewo area were similar in terms of their petrography and reservoir properties, and the area was named "Region 1" (Fig. 1). The area of Siekierki - Kromolice - Miłosław showed slight changeability in petrography of the eolian sandstones, but significant variability of their reservoir properties (Buniak et al. 2009). 


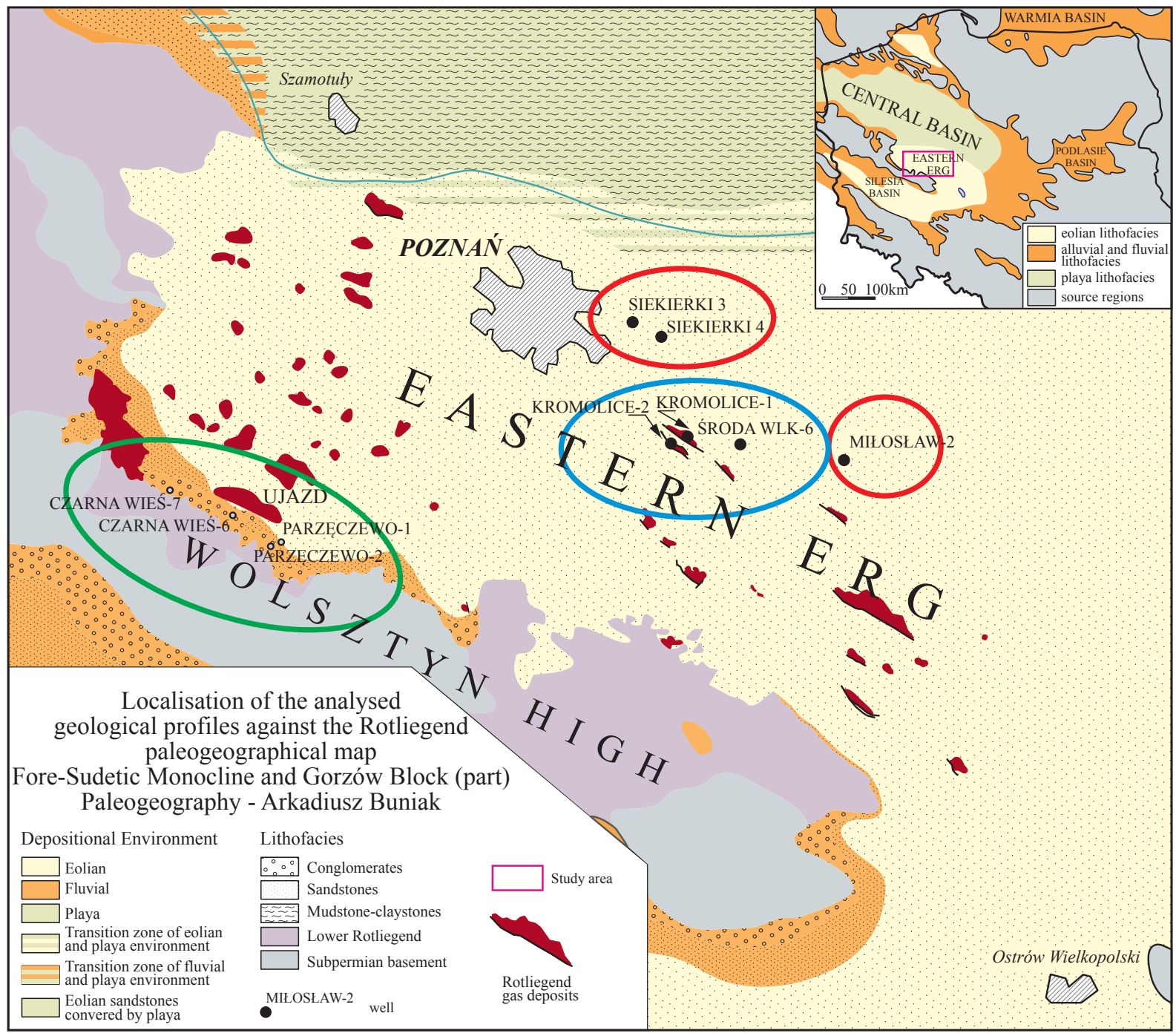

Fig. 1. Paleogeographic map of the Upper Rotliegend, with wells in the study location (acc. to Buniak 2008b): green ellipse Region 1, blue ellipse - Region 2, red ellipses - Region 3

Due to this feature, the area was divided into two regions. The first one, with good reservoir properties, including wells Kromolice-1 and Kromolice-2, Środa Wielkopolska-6 was named "Region 2". The other one, with low reservoir properties, including wells Siekierki-3, Siekierki-4, and Miłosław-2 was called "Region 3". Tight gas occurs in the region with poor reservoir properties (Kiersnowski et al. 2010).

\section{Czarna Wieś - Parzęczewo area}

In the Czarna Wieś - Parzęczewo area, the Rotliegend deposits pinched out to the Wolsztyn High (Fig. 1). Geological profiles show that the Rotliegend sediments varied in lithology, facies development and reservoir properties. Logs and results of the comprehensive interpretation in the selected wells (Fig. 2) illustrate the variability. The collateral changes of the sedimentary facies were caused by the tectonics in the marginal zone of the basin, as well as by paleoclimatic changes (Buniak et al. 2008b). The eolian sandstones from wells CW-5, CW-6, CW-7, P-1, and P-2 were selected for the study. The Upper Rotliegend profile of the Czarna Wieś - Parzęczewo study area consisted of sandstones developed in the dune and interdune eolian facies and sandstones and conglomeratic sandstones and conglomerates of fluvial and alluvial facies (Fig. 2).

\section{Siekierki - Kromolice - Miłosław area}

The Siekierki - Kromolice - Miłosław area was located in the northern part of the Fore-Sudetic Monocline, near the boundary of the Mogilno-Łódź Synclinorium (Fig. 1). 


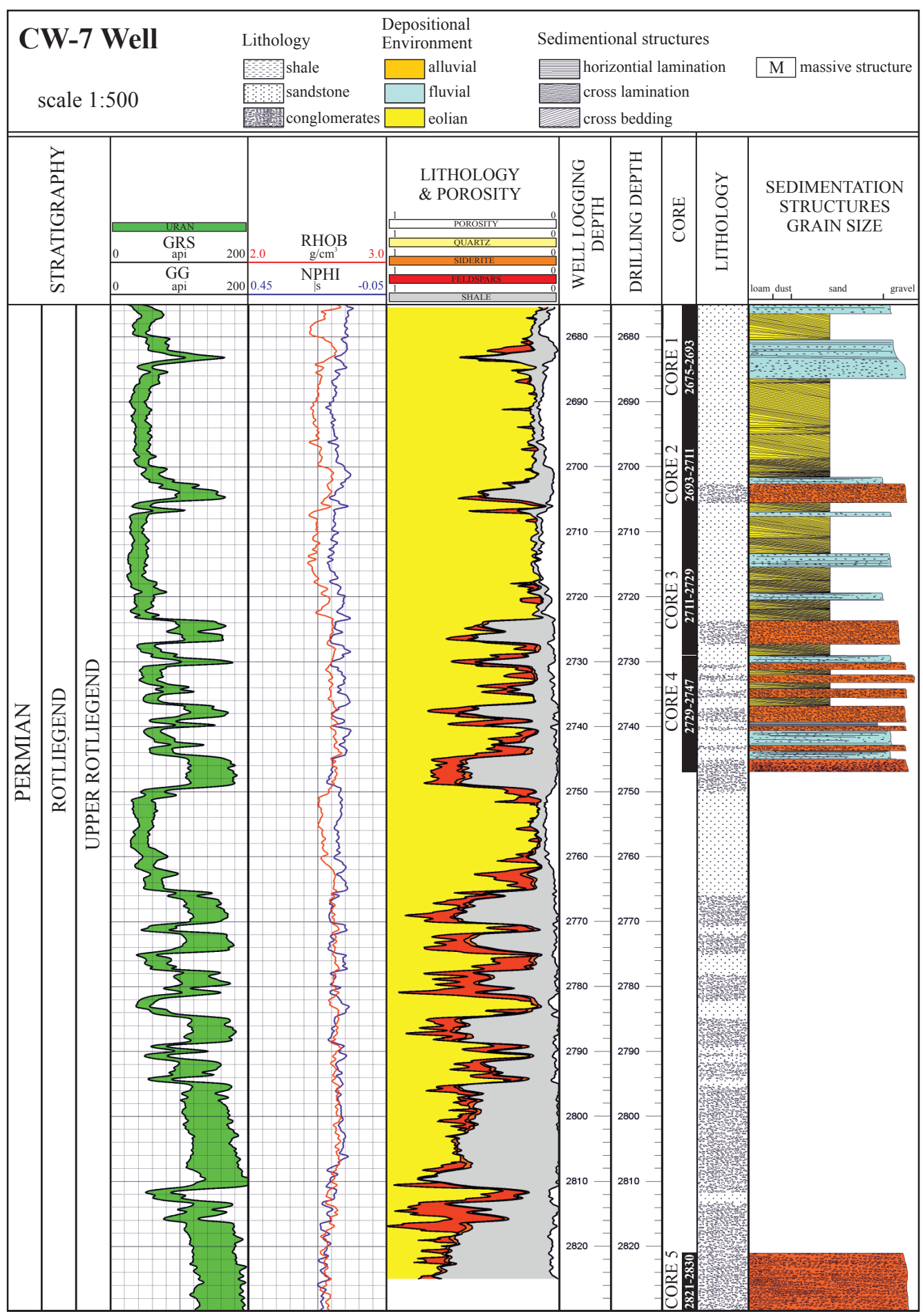

Fig. 2. Logs and results of the comprehensive interpretation in the Czarna Wieś-7 well in Region 1 (acc. to Kozłowski \& Wolański (POGC) interpretation). Results of sedimentological analyses acc. to Buniak et al. (2008b)

In that area, the top of the Rotliegend deposits was at a depth of $3.5-3.7 \mathrm{~km}$. Only the wells SW-4, SW-5, WG-1, K-1 and K-2 proved to have small gas deposits in structural traps in the top of the Upper Rotliegend (Buniak et al. 2008a). Many other wells, with slight gas inflows, had been abandoned, due to the very poor reservoir properties of the rocks. Of great importance was the Aurelian success at the Siekierki structure, until then considered as non-prospective and rediscovered by well Trzek-1 (San Leon Energy, on-line). 
The eolian sandstones from wells SW-6, K-1, $\mathrm{K}-2, \mathrm{~S}-3, \mathrm{~S}-4$, and M-2 were selected for this study. Logs and results of the comprehensive interpretation in the selected wells (Fig. 3) illustrate the variability of lithology and facies development and reservoir properties. The Upper Rotliegend profile of the Siekierki - Kromolice - Miłosław area was built of sandstones of dune and interdune eolian facies. On the other hand, its top consists of the sandstones of shallow-water environment (redeposited beds) (Fig. 3) (Buniak et al. 2009). Depths and thicknesses of the Upper Rotliegend deposits in the selected wells are presented in Table 1.

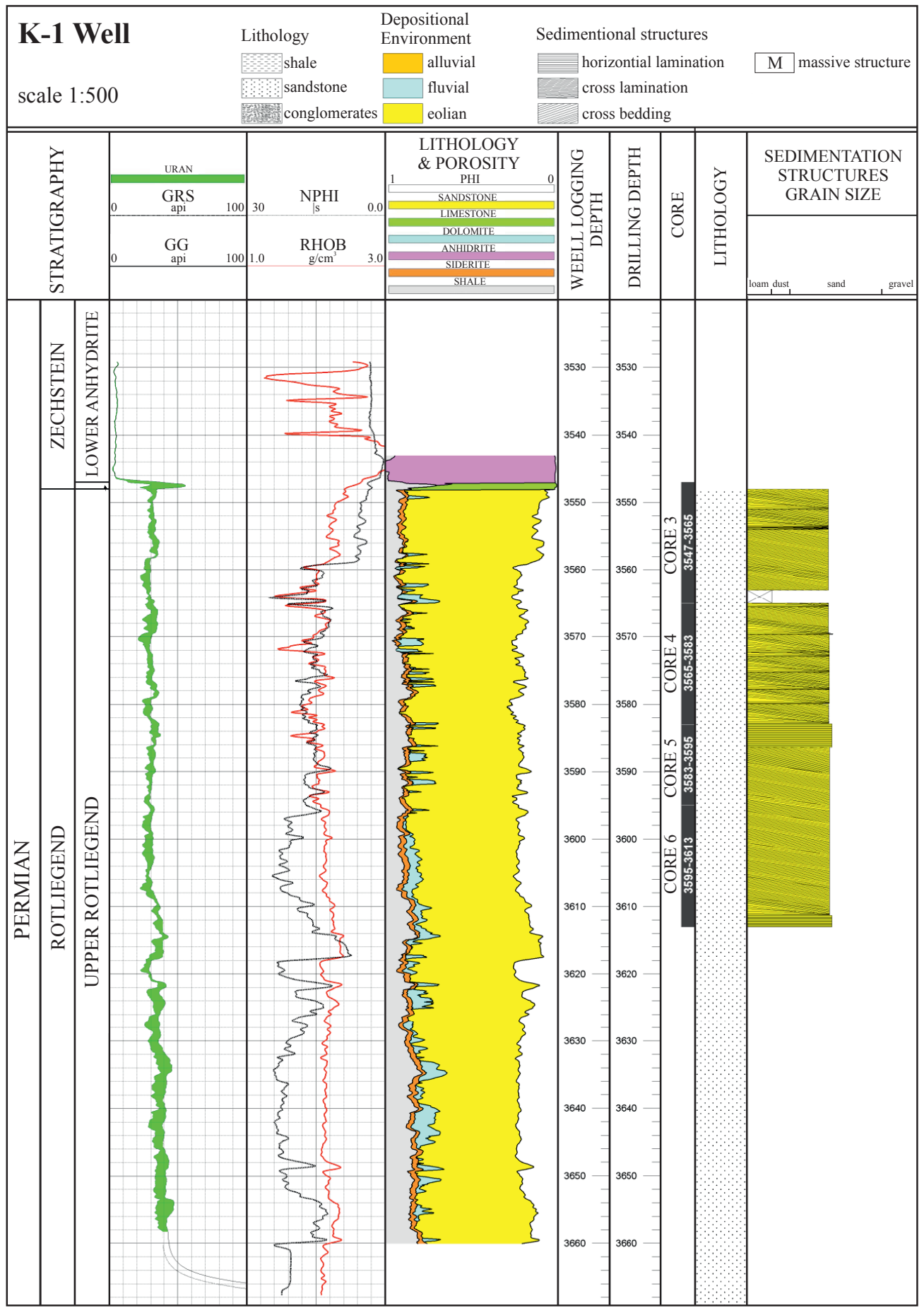

Fig. 3. Logs and results of the comprehensive interpretation in the Kromolice-1 well in Region 2 (acc. to Kozłowski \& Wolański (POGC) interpretation). Results of sedimentological analyses acc. to Buniak et al. (2008b) 
Table 1

Depth and thickness of the Upper Rotliegend deposits acc. to well logging interpretation (reports for wells CW-6, CW-7, P-1, P-2, $S W-6, K-1, K-2, S-3, S-4, M-2)$

\begin{tabular}{|c|c|c|}
\hline \multirow[t]{2}{*}{ Well } & $\begin{array}{l}\text { Sedimentary series of the UpperRotliegend } \\
\text { (depth acc. to well logging) }\end{array}$ & $\begin{array}{l}\text { Thickness of the Upper } \\
\text { Rotliegend rocks }\end{array}$ \\
\hline & {$[\mathbf{m}]$} & {$[\mathbf{m}]$} \\
\hline Czarna Wieś-6, CW-6 & $2655.6-2801.0$ & $>145.4$ \\
\hline Czarna Wieś-7, CW-7 & $2675.0-2830.0$ & $>155.0$ \\
\hline Parzęczewo-1, P-1 & $2613.0-2871.0$ & 258.0 \\
\hline Parzęczewo-2, P-2 & $2559.5-2737.0$ & $>177.5$ \\
\hline Środa Wielkopolska-6, SW-6 & $3709.5-3742.0$ & $>32.5$ \\
\hline Kromolice-1, K-1 & $3548.0-3668.0$ & $>120.0$ \\
\hline Kromolice-2, K-2 & $3545.0-3661.0$ & $>116.0$ \\
\hline Siekierki-3, S-3 & $3650.5-4085.5$ & 435.0 \\
\hline Siekierki-4, S-4 & $3668.5-4099.0$ & 430.5 \\
\hline Miłosław-2, M-2 & $3697.0-4240.0$ & 543.0 \\
\hline
\end{tabular}

\section{DATA SET}

Preliminary laboratory studies of samples included measurement of total porosity by means of helium pycnometry, absolute permeability using nitrogen, and mineral composition by means of X-ray diffraction (XRD). The data set contained 19 samples from the Czarna Wieś - Parzęczewo (Region 1) and 21 samples from each of the two other regions (Region 2 and Region 3). The samples were selected so that each region was represented by diverse porosity and permeability. Total porosity of rock samples from wells CW-5, CW-6 and $\mathrm{CW}-7$ and P-1 and P-2 (Region 1) ranged from $2 \%$ to $17 \%$, with an average value of $10.5 \%$. The absolute permeability fell within the range of $0.03-127 \mathrm{mD}$ with a geometric mean equal to $1.7 \mathrm{mD}$. Samples from wells SW-6 and K-1 and K-2 (Region 2) had porosity of 10-21\%, at an average value of $15.4 \%$. Permeability range was 0.39 $253.67 \mathrm{mD}$, with a geometric mean of $10.3 \mathrm{mD}$. Samples from wells S-3 and S-4 and M-2 (Region 3) had a porosity of $7-20 \%$, with an average value of $11.7 \%$. Permeability ranged from $0.02 \mathrm{mD}$ to $5.78 \mathrm{mD}$, with a geometric mean of $0.19 \mathrm{mD}$. The samples differed significantly in their porosity and permeability values (Fig. 4). Three big markers in Figure 4 represent calculated average values for each region. Samples from the Region 2 had the biggest average porosity (15.4\%) and absolute permeability $(10.3 \mathrm{mD})$.

In all samples, the skeleton was found to be built mainly of quartz grains. The other major component included lithoclasts of igneous rocks (represented by various granitoids and effusive rocks), metamorphic rocks (shales and quartzites) and sedimentary rocks (claystones, less often mudstones). The next constituent of the framework included K-feldspars and plagioclases. Carbonate and clay were the main cements of the arenites in question. The carbonate cement (calcite) has been found in all samples. Usually, it filled the pores. The volume of clayey cement ranged from $0.6 \%$ to $16.1 \%$. It occurred as thin coats around grains or filled the entire pore space. Quartz cement was observed occasionally as pore space filling and, rarely, as coats. Clayey-ferruginous matrix (up to $1.15 \%$ volume) was seldom noted.

The mineral composition of most samples was determined with the use of the X-ray diffraction method. Three representative diffraction patterns are presented in Figures 5-7. They are related to samples from selected wells in the Regions 1, 2 and 3. Collective characteristics of mineral composition as result of the X-Ray diffraction are presented below. 


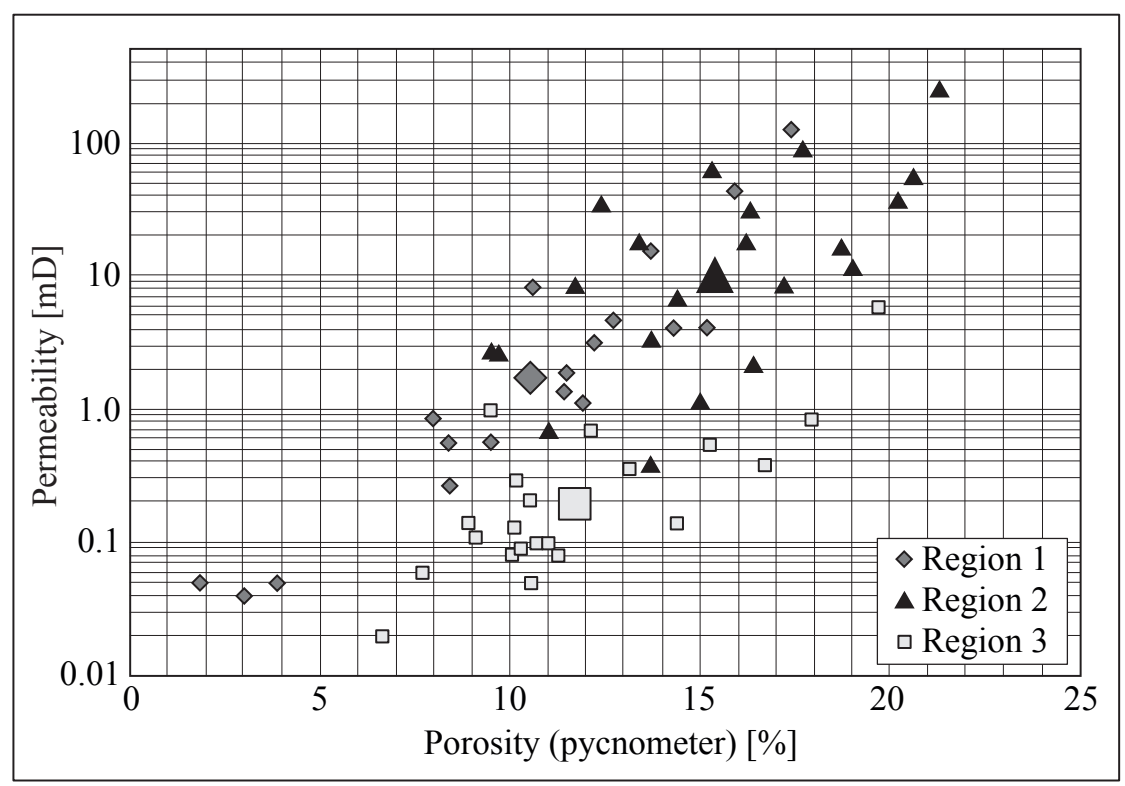

Fig. 4. Absolute permeability vs. total porosity; big markers mean average values for three selected regions

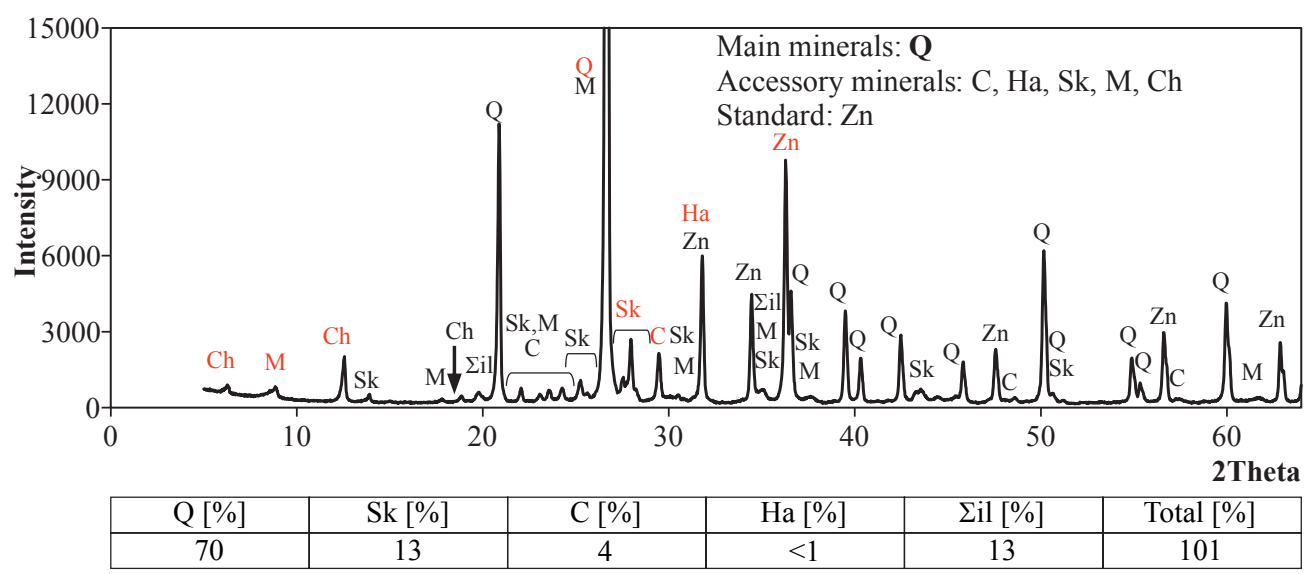

Fig. 5. Diffraction pattern in the Czarna Wieś-7 well, Region 1, depth of core 2689.05 m (Q - quartz, 33-1161; C - calcite, 5-586; Ha - halite, 5-628; Sk-feldspars, group identification; $M$ - micas and illite type minerals; Ch - chlorite; Dil - sum of clay minerals; $\mathrm{Zn}$ - zincite, 36-1451 - standard)

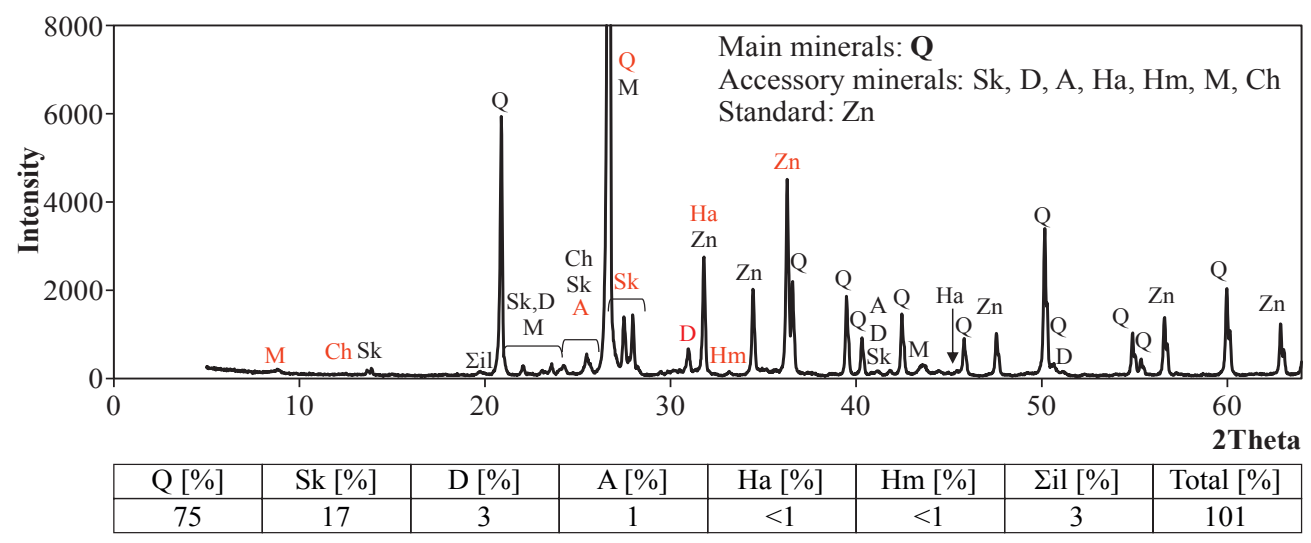

Fig. 6. Diffraction pattern in the Kromolice-2 well, Region 2, depth of core: 3593,60 m (Q - quartz, 33-1161; D - dolomite, 36-426; A - anhydrite, 37-1496; Ha - halite, 5-628; Hm - hematite, 33-664; Sk-feldspars, group identification; M - micas and illite type minerals; Ch-chlorite; Sil - sum of clay minerals; $\mathrm{Zn}$ - zincite, 36-1451 - standard) 


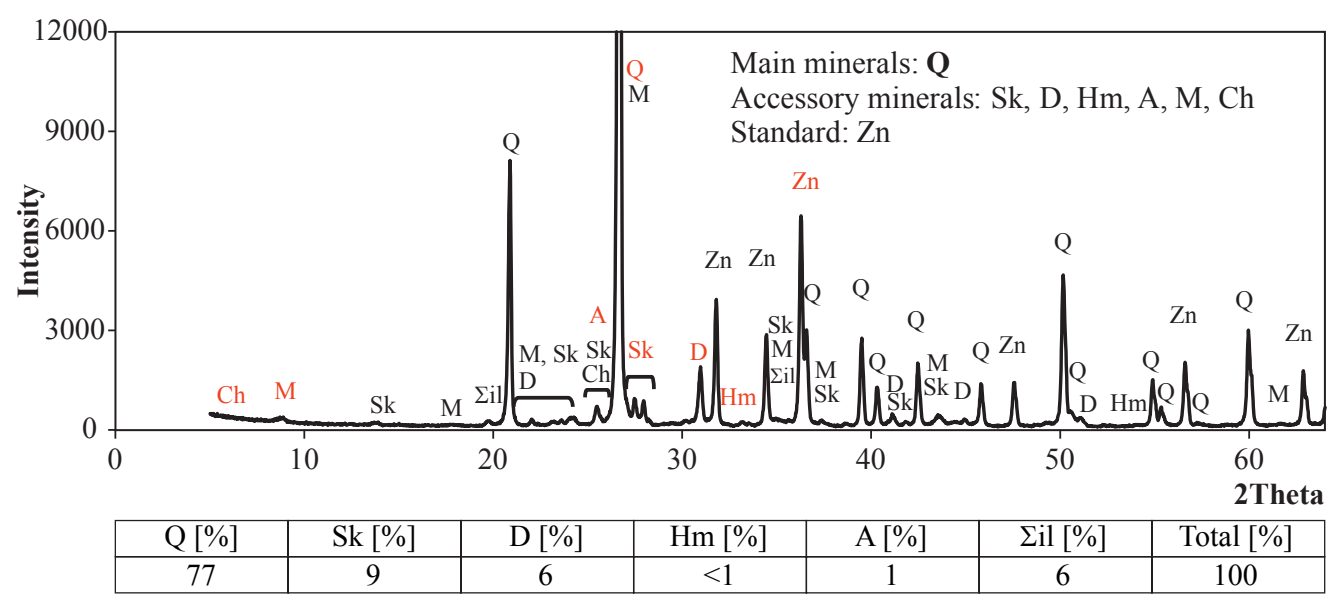

Fig. 7. Diffraction pattern in the Miłosław-2 well, Region 3, depth of core: $3737,35 \mathrm{~m}$ (Q-quartz, 33-1161; D - dolomite, 36-426; Hm - hematite, 33-664; A - anhydrite; Sk-feldspars, group identification; $M$ - micas and illite type minerals; Ch-chlorite; Eil-sum of clay minerals; Zn-zincite, 36-1451-standard)

Quartz was the dominant mineral in all samples. The smallest average amount (ca. 68\%) was observed in samples from wells of Region 1. In wells of Region 2, it amounted to 75\%, while in Region 3 , it came up to $77 \%$. The largest contribution of clay minerals (14\%) was found in the samples of Region 1, then of Region 3 (8\%) and, finally, Region 2 (4\%). Feldspar content was highest in samples of Region 2. In the other two regions, it amounted to about $10 \%$. The amount of dolomite in the samples from Regions 2 and 3 was evaluated at a few percent, while its content, in most samples from Region 1, was inappreciable. Calcite was found in great abundance (1-31\%) only in the samples from Region 1. Other minerals, i.e. halite, anhydrite and hematite were recognized in negligible amounts.

Results of other analyses available from the Oil and Gas Institute, Krakow, Poland, were also considered in this research. Some results are cited here to make better geological descriptions of the study material (Dohnalik \& Zalewska 2008, Zalewska et al. 2009). Sandstones of the discussed facies were more abundant in geological profiles of either area. They were fine-grained to medium-grained, occasionally coarse-grained. Locally, they were well and very well sorted and had bimodal grain size distribution. A characteristic feature was cross lamination, which was accentuated by laminas with normal or reverse grain size gradation thin, dark brown, very fine-grained laminas and shaly laminas. Further up, dune core sandstones passed into dune top sandstones. Dune core sandstones were encountered in all wells in question. Petrologic analyses were made using 27 thin sections. The observations focused on mineral composition of the skeleton, type and development of cement, and the shape and size of grains and the mutual relationship between grains were from the results of the unpublished data (Ziemianin 2012). As a result of petrologic studies, it was found that analysed rocks were fine-grained and very fine-grained sublithic arenites, litharenites and subarkoses. The grains were usually mediumto well-graded or well- and very well-graded, sharpedged and well-rounded or very-well-rounded. The structure was usually notoriented however sometimes laminas occurred, built of medium-grained material, within very fine-grained rocks.

\section{METHODOLOGY}

Computed X-ray microtomography was the main technique used in this research. The results were compared with outcomes of the other laboratory methods: mercury injection capillary pressure, nuclear magnetic resonance experiment, helium pycnometry and permeability measurements.

\section{Computed X-ray microtomography (micro-CT)}

During the micro-CT measurement X-ray tube emitted photons that penetrated a sample. Due to the inhomogeneous structure of the sample, the radiation reaching the detector surface was attenuated to different degrees. The detector changed the X-ray radiation into an electric signal, which was next saved as digital X-ray images called 
projections. The sample can be fully rotated during the scan but was stopped by user-defined rotation angle. The detector registered the projection at each step. Exemplary projections of the Rotliegend sandstone sample (Fig. 8) showed the micro-CT capabilities to visualize a diversified image of rock.
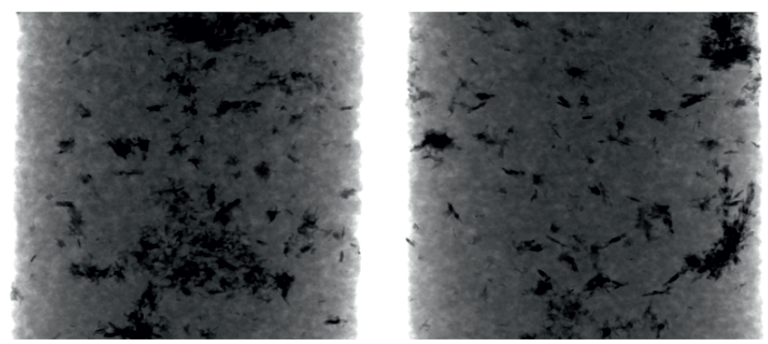

Fig. 8. Exemplary projections of the Rotliegend sandstone sample; bright grey - pores, gray - mineral skeleton, black dense minerals (result of authors' own work)

The next stage of the micro-CT data processing was the reconstruction of the object's spatial image based on saved projections. Prior to computer analysis, the image should be binarized (Cnudde 2005). The image binarization was a process, in which an image was converted from a number of gray levels into a two-color binary image, typically black and white. In the case of rock samples, binarization separates voxels representing mineral skeleton and porous space from the sample volume (Fig. 9). Voxel, a conventional cubic unit of volume, was used in the micro-CT data analysis. Voxel size decided on the micro-CT method resolution (Golab et al. 2013). A gray-scale cross-section, through a sandstone sample is shown in Figure 9A. The gray part corresponds to the skeleton, while the black one to the pore structure. Figure 9B presents a binary image of the sample from Figure 9A; white corresponds to the pore space and black - to the rock skeleton (Dohnalik et al. 2009).
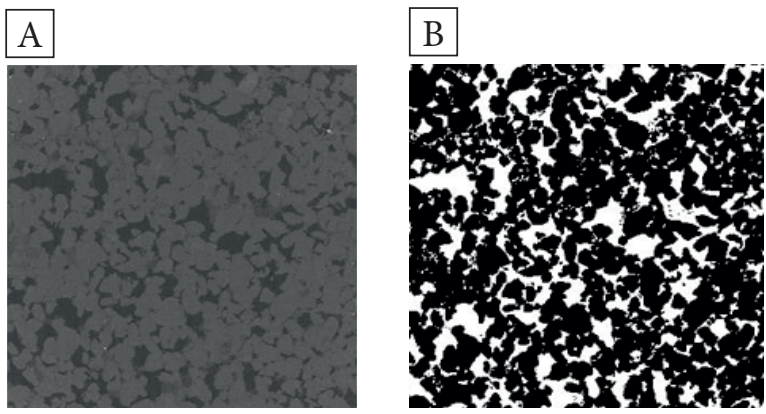

Fig. 9. Cross-section through a sandstone sample: A) before binarization; B) after binarization (Dohnalik et al. 2009)
Porosity calculation. After binarization the porosity coefficient $\left(K_{p}\right)$ was calculated based on equation:

$$
K_{p}=V_{p} /\left(V_{p}+V_{s}\right)
$$

where $V_{p}$ is voxel volume representing pore structure, and $V_{s}$ is voxel volume representing the skeleton.

Parameters characterizing the pore structure, i.e. porosity and coefficient of homogeneity of pore structure were determined using local porosity examination, box counting dimension, mean chord length, normalized Euler number and coordination number. The precise definitions of the above mentioned parameters are presented in the next chapters. Below, short primary information is provided to make easier reading of the next chapters. The measure of the pore structure homogeneity was the value of the relative standard deviation, calculated on the basis of standard deviation of local porosity for a small subsample and porosity of the large sample (section: Homogeneity of pore structure distribution). Box counting dimension was calculated with the use of an algorithm, which was similar to the standard box counting measurement for 2D images (section: Box counting dimension of pore structure). Mean chord length was defined as the mean length of chords that were drawn through the selected layer of pores (section: Mean chord length through the pore layer). The Euler number was calculated as a parameter describing the communication of spaces of irregular shape (i.e. fractures, channels with roots, cavities in soil made by worms). Normalized Euler numbers were obtained by dividing the calculated Euler numbers by the number of all selected objects (section: Euler number). The Coordination number was determined using the number of pore channels, end points, and pores defined on the basis of medial axis transformation of pore space image (section: Medial axis transformation of pore space image).

Separation of unconnected objects. To obtain extra information on the pore structure image all isolated objects (pore subsystems) were separated by analysing each voxel neighbourhood. Communicated voxels, which belonged to the neighbourhood of a studied voxel, fulfilled a condition defined by Fisher et al. (2003). The computer 
program verified whether the condition of belonging to the neighbourhood had been fulfilled. The neighbourhood of 26 voxels was examined to distinguish objects separated from the examined object. A graphical illustration of the analysis of voxel neighbourhood is shown in Figure 10 - pores are marked with green (Fig. 10A), while unconnected objects are marked with different colors (Fig. 10B).
A

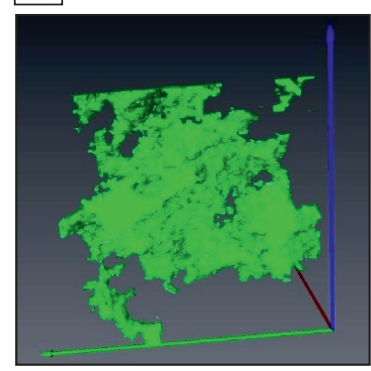

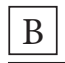

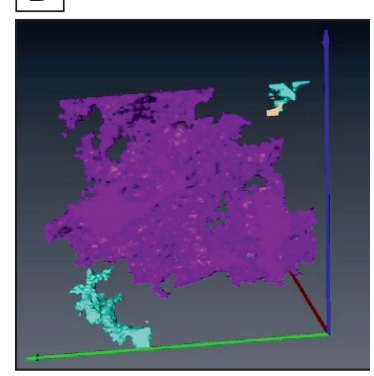

Fig. 10. Microtomographic image of porous rock: A) porous structure; B) unconnected objects (result of authors' own work)

Volume classification. During volume classification, each separated object obtained its order number and was marked with a color or grayscale. Further analysis provided information on the volume of each object. The applied software (MAVI) enabled the filtration of a 3D image on the basis of the objects' volumes and visualized pore sub-types from a selected volume range. The MAVI function was applied to classify objects on the basis of their volume and visualize their spatial distribution. The principle of classifying pore subsystems is presented in Table 2.

Tortuosity of pore channels. The tortuosity was defined as a geometric property of a path of communicated points (pixels in 2D image, voxels in 3D volume); depending on a number of turns in the path (Fig. 11). Tortuosity was calculated as the ratio of the length of the path between two points to the distance between them:

$$
t_{i j}=l_{j} / l_{i}
$$

where $l_{j}$ is a path length between two points, and $l_{i}$ is a distance between two points.

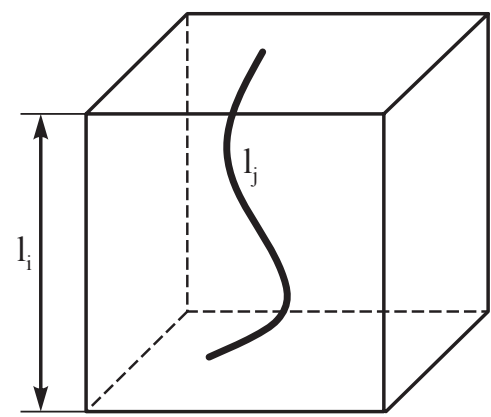

Fig. 11. Tortuous pore path

Homogeneity of pore structure distribution. A sample was divided into smaller cubes and then so-called local porosity of subsamples was evaluated. Analysis of local porosity of input images, with the size of $900 \times 900 \times 400$ voxels, was made for subsamples, with the size of $100^{3}$ voxels. As such, 324 values for local porosity were obtained for each sample. The measure of the pore structure homogeneity was the value of the relative standard deviation $\left(\sigma_{r}\right)$, given by equation:

$$
\sigma_{r}=\sigma_{100} / K_{p \mathrm{CT}}
$$

where $\sigma_{100}$ is the standard deviation of local porosity for a subsample $100 \times 100 \times 100$ voxels, and $K_{p \text { Ст }}$ is porosity for the image $900 \times 900 \times 400$ voxels.

Table 2

Volume of distinguished objects

\begin{tabular}{|l|c|c|c|c|c|c|c|}
\hline $\begin{array}{l}\text { Volume of pore } \\
\text { sub-system [voxel] }\end{array}$ & $1-9$ & $10-99$ & $100-999$ & $1000-9999$ & $10000-99999$ & $\begin{array}{c}100000- \\
-1000\end{array}$ & $>1000$ \\
\hline $\begin{array}{l}\text { Volume of pore } \\
\text { sub-system }\left[\mu \mathrm{m}^{3}\right]\end{array}$ & $2 \cdot 10^{2}-2 \cdot 10^{3}$ & $2 \cdot 10^{3}-2 \cdot 10^{4}$ & $2 \cdot 10^{4}-2 \cdot 10^{5}$ & $2 \cdot 10^{5}-2 \cdot 10^{6}$ & $2 \cdot 10^{6}-2 \cdot 10^{7}$ & $2 \cdot 10^{7}-2 \cdot 10^{8}$ & $>2 \cdot 10^{8}$ \\
\hline Class & I & II & III & IV & V & VI & VII \\
\hline Colour & yellow & blue & red & green & white & violet & rose \\
\hline
\end{tabular}


To determine a boundary value below which porosity distribution is assumed to be homogeneous, tests were made on samples of the white sandstone from Szydłowiec. On the basis of a number of examinations, the Oil and Gas Institute considered that sandstone as a standard material with homogeneous pore structure. In this paper, it was assumed that samples of the Rotliegend sandstone have homogeneous pore structure if the value of relative standard deviation does not exceed 0.3.

Box counting dimension of pore structure. Box counting dimension was calculated with the use of an algorithm that was similar to the standard box counting measurement for 2D images (Peitgen et al. 1995). Images with the size of $900 \times$ $900 \times 400$ voxels were analyzed. A grid with cells equal to cubes defined by the user was superimposed on the input spatial image. Then it was calculated how many cubes contained voxels related with the pore layer. Next, the grid cell size was reduced. The calculations were stopped when the grid cell reached the defined minimal size. The result of calculations was a straight line drawn as a logarithm of cell count vs. logarithm of cell size (Fig. 12).

Mean chord length through the pore layer. The mean cord length was an important parameter calculated from the microtomography images. It was defined as the mean length of chords that were drawn through the layer of pores (Młynarczuk 2004, Osher \& Schladitz 2009). The relationship between the size of pore subsystems and the mean chord length for an examined sample was used as a measure of pore structure (Tab. 3).

It can be noted that the mean cord length rose significantly with increasing volume of pore subsystems. Hence, samples with well-developed pore structure had high values of that parameter.

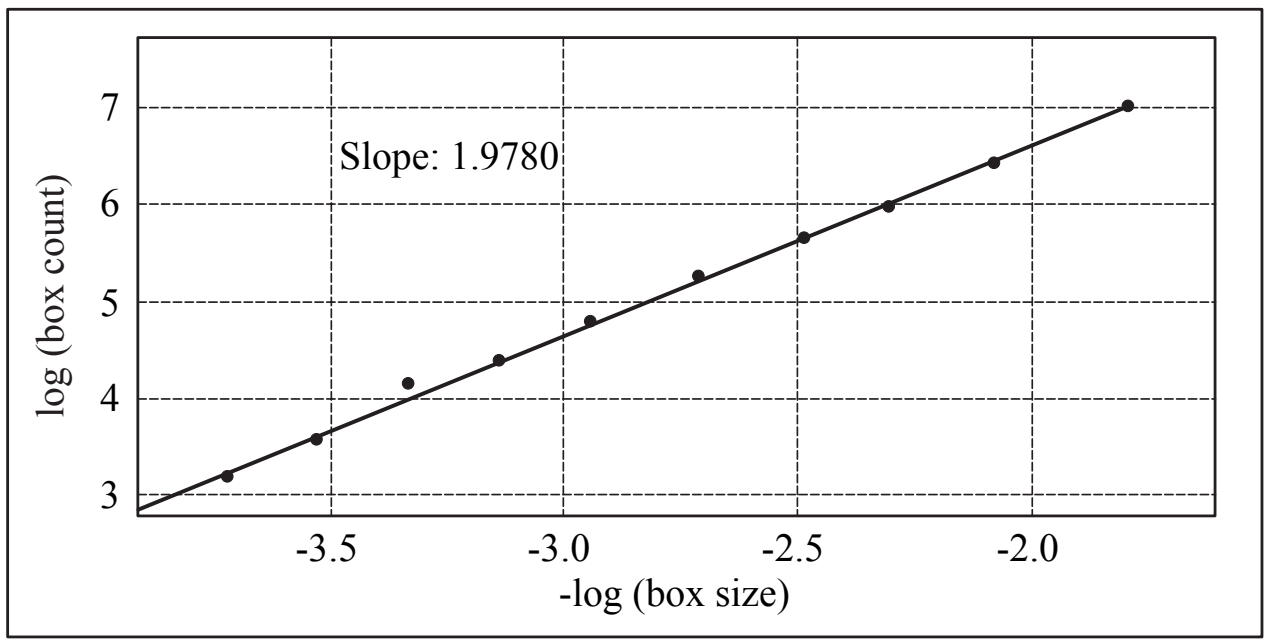

Fig. 12. Box counting dimension of analyzed pore structure; slope of regression line

Table 3

Relationship between mean chord length and volume of pores

\begin{tabular}{|c|c||c|c|}
\hline $\begin{array}{c}\text { Volume of analyzed pores } \\
\text { [voxel] }\end{array}$ & $\begin{array}{c}\text { Mean chord length } \\
\text { [voxel] }\end{array}$ & $\begin{array}{c}\text { Volume of analyzed pores } \\
\text { [voxel] }\end{array}$ & $\begin{array}{c}\text { Mean chord length } \\
\text { [voxel] }\end{array}$ \\
\hline$<10$ & 1.8 & $<10$ & 1.80 \\
\hline $10-100$ & 2.1 & $<100$ & 2.00 \\
\hline $100-1000$ & 3.2 & $<1000$ & 2.26 \\
\hline $1000-10000$ & 4.6 & $<10000$ & 2.34 \\
\hline$>10000$ & 5.3 & $<100000$ & 4.99 \\
\hline
\end{tabular}


Euler number. Rock and soil studies showed that in addition to number and size of pores, it was important to know how the pores are interconnected (Toriwaki \& Yonekura 2002). The Euler number was calculated, as a parameter describing the communication of spaces of irregular shape (i.e. fractures, channels with roots, cavities in soil made by worms) (Vogel 2002). The Euler number is defined by:

$$
\chi=N-C+H
$$

where $N$ is a number of isolated pores, $C$ is a number of connections, and $H$ is a number of skeleton grains totally surrounded by void.

The value of $H$ was practically negligible for rock images because in the nature there are no particles of rock skeleton that are surrounded by air. Therefore, it was true that for positive values of $\chi$, i.e. $N>C$, the pore structure was poorly connected, while for $C>N$, the pore structure had many communications. Results of the Euler number analysis for image $200 \times 200 \times 200$ voxels with calculated total porosity of $22 \%$ (sample 9225, SW-6, Region 2) are presented in Table 4.

\section{Table 4}

Relationship between Euler number and volume of analyzed objects

\begin{tabular}{|c|c|c|}
\hline $\begin{array}{c}\text { Volume } \\
\text { of analysed pores } \\
\text { [voxel] }\end{array}$ & $\begin{array}{c}\text { Number } \\
\text { of analysed } \\
\text { isolated objects }\end{array}$ & $\begin{array}{c}\text { Euler } \\
\text { number }\end{array}$ \\
\hline$<10$ & 3562 & 3425 \\
\hline 100 & 6439 & 6015 \\
\hline 1000 & 6467 & 5969 \\
\hline 10000 & 6468 & 5962 \\
\hline all & 6469 & -5903 \\
\hline
\end{tabular}

There was an evident dependence between the number of isolated objects in the pore space and the Euler number. The Euler number rose, when the pore volume in an isolated object increased from 10 to 100 voxels, and the number of objects (pores) grew. When the pore volume increased to 10000 voxels, the difference was small for an almost constant number of analyzed objects. Then, the Euler number decreased, because there appeared interconnected pore systems. The minimal value of -5903 was calculated for the full image of the structure. Normalized Euler numbers were obtained by dividing calculated Euler numbers by the number of all selected objects (Tabs 5-8).

\section{Medial axis transformation of pore space image} (calculation of coordination number for the pore structure). Reducing the spatial image to medial axis allowed us to construct the geometry and topology of the original structure to be preserved (Yang 2005). The medial axis transformation consisted in isotropic cutting-out ('erosion') of white voxels, over the whole area of image elements (Dong 2008). Points at which the cutting-out planes contacted were included into the sample image (Fig. 13).
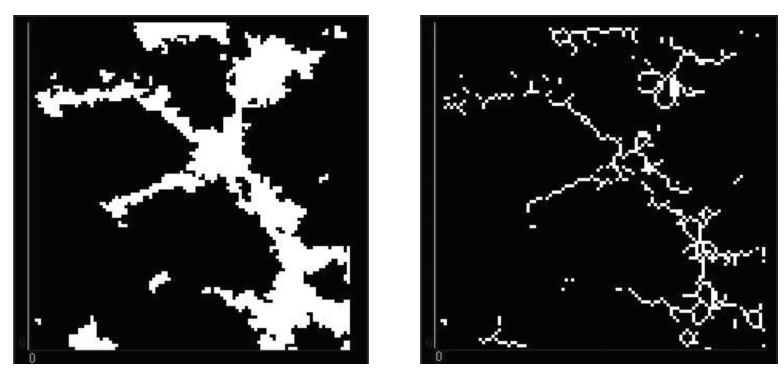

Fig. 13. Exemplary medial axis transformation (result of authors'own work)

After medial axis transformation, the following elements were discriminated in the image: end points - voxels located at the end of a single branch, nodes (pores) - voxels constituting intersection of two branches, and branches (pore channels) - each sequence of voxels that ends with intersection or node. The elements were defined on the basis of analysis of the neighborhood of the study point. The application of results of medial axis analysis generated an image with particular elements of skeleton.

To obtain the medial axis of the pore space, in this paper, a function that generated the topology tree of the analyzed structure (Sato et al. 2000) was applied. This approach differed from a typical medial axis transformation in that it did not generate a loop. Having determined the number of pore channels, end points, and pores, we calculated the coordination number for samples based on equation:

$$
\bar{L}_{K}=\frac{2 \cdot L_{k}-L_{Z}}{L_{W}}
$$

where $L_{K}$ is a number of channels, $L_{Z}$ is a number of end points, and $L_{W}$ is a number of nodes. 


\begin{tabular}{|c|c|c|c|c|c|c|c|c|c|c|c|c|c|c|c|c|c|}
\hline \multirow{9}{*}{ 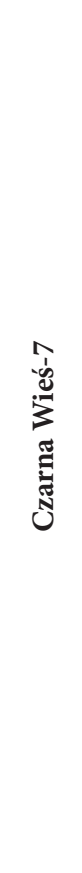 } & ळे & $\stackrel{\infty}{\stackrel{\infty}{\sim}}$ & $\underset{\stackrel{\overbrace{}}{\mathrm{i}}}{ }$ & $\begin{array}{c}\text { } \\
\stackrel{+}{+}\end{array}$ & 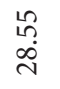 & $\stackrel{\varrho}{\leftrightarrows}$ & 0 & 0 & $\stackrel{\text { ڤ }}{\rightarrow}$ & సે & $\begin{array}{l}\stackrel{\bullet}{0} \\
i\end{array}$ & $\stackrel{n}{\underset{H}{*}}$ & ڤે. & 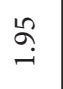 & ڤे & 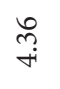 & $\underset{+}{\stackrel{\leftrightarrow}{+}}$ \\
\hline & ळ̊ & $\stackrel{\hat{m}}{+}$ & $\begin{array}{l}\hat{a} \\
\dot{g}\end{array}$ & 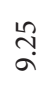 & $\stackrel{m}{=}$ & $\stackrel{\mathbb{N}}{N}$ & $\stackrel{n}{\stackrel{n}{N}}$ & $\begin{array}{l}\stackrel{m}{\dot{b}} \\
\dot{q}\end{array}$ & $\stackrel{\widehat{\hat{b}}}{\hat{b}}$ & $\tilde{0}$ & $\stackrel{\infty}{i}$ & $\begin{array}{l}\vec{\infty} \\
\stackrel{-}{\triangle}\end{array}$ & $\stackrel{\widetilde{n}}{0}$ & $\stackrel{\sim}{i}$ & $\overrightarrow{\dot{m}}$ & $\stackrel{\ddot{r}}{\ddot{n}}$ & 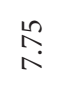 \\
\hline & ڤ̆ & $\begin{array}{l}\infty \\
\dot{r}\end{array}$ & $\begin{array}{l}\overrightarrow{i n} \\
\dot{I}\end{array}$ & $\begin{array}{l}\vec{\infty} \\
\dot{m}\end{array}$ & 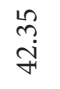 & $\stackrel{\text { f }}{+}$ & 0 & 0 & $\stackrel{\hat{\sigma}}{-}$ & fै & $\stackrel{\tilde{n}}{i}$ & $\begin{array}{l}\text { L } \\
\stackrel{0}{0} \\
\end{array}$ & $\stackrel{\mathscr{n}}{\tilde{O}}$ & $\begin{array}{l}\stackrel{\infty}{\infty} \\
\stackrel{-}{-}\end{array}$ & $\begin{array}{l}\tilde{N} \\
\stackrel{n}{m}\end{array}$ & $\vec{m}$ & 点 \\
\hline & $\stackrel{\text { ळ̊ }}{\circ}$ & $\underset{i}{\stackrel{i}{i}}$ & 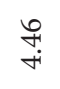 & : & $\begin{array}{l}\infty \\
\stackrel{\infty}{\Xi} \\
\end{array}$ & $\begin{array}{l}\vec{\infty} \\
\stackrel{\Theta}{\sim}\end{array}$ & $\stackrel{尺}{\stackrel{1}{\wedge}}$ & ले & $\stackrel{m}{n}$ & $\stackrel{l}{\sharp}$ & $\begin{array}{l}\infty \\
\stackrel{\infty}{i}\end{array}$ & $\underset{\widetilde{N}}{\stackrel{\sim}{*}}$ & $\stackrel{\infty}{\infty}_{\substack{i \\
i}}$ & $\underset{i}{\stackrel{H}{i}}$ & $\underset{m}{\stackrel{H}{m}}$ & $\begin{array}{l}\hat{\sigma} \\
\stackrel{i}{1}\end{array}$ & $\underset{+}{\stackrel{+}{+}}$ \\
\hline & ڤ్ & $\begin{array}{l}\stackrel{\infty}{0} \\
\text { in }\end{array}$ & 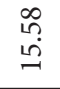 & $\begin{array}{l}\infty \\
\stackrel{\infty}{\circ}\end{array}$ & $\begin{array}{l}\widetilde{\sigma} \\
\stackrel{-}{0}\end{array}$ & ને & $\stackrel{\leftrightarrow}{\ddot{m}}$ & $\begin{array}{l}\mathscr{n} \\
\stackrel{F}{\gamma}\end{array}$ & $\stackrel{\infty}{\stackrel{\infty}{N}}$ & กี & $\begin{array}{l}\stackrel{\sim}{\infty} \\
\stackrel{i}{i}\end{array}$ & $\begin{array}{l}\stackrel{9}{n} \\
\stackrel{n}{n}\end{array}$ & $\begin{array}{l}+ \\
0 \\
i\end{array}$ & $\stackrel{\text { oे }}{\text { in }}$ & $\stackrel{\stackrel{\sim}{\sim}}{+}$ & 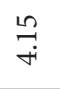 & iे \\
\hline & ळे & 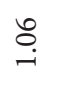 & 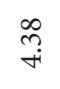 & $\begin{array}{l}\stackrel{L}{0} \\
\stackrel{]}{\mathrm{I}}\end{array}$ & $\stackrel{\stackrel{\sim}{\sim}}{\underset{\sim}{\sim}}$ & $\begin{array}{l}\overrightarrow{\mathrm{N}} \\
\overrightarrow{\mathrm{i}}\end{array}$ & $\stackrel{\sqrt[n]{n}}{\underline{n}}$ & $\begin{array}{l}\infty \\
\stackrel{\infty}{\triangle}\end{array}$ & $\begin{array}{l}\stackrel{\leftrightarrow}{+} \\
\stackrel{+}{+}\end{array}$ & $\overrightarrow{\tilde{\sigma}}$ & $\begin{array}{l}\hat{b} \\
\vec{i}\end{array}$ & $\stackrel{\sim}{\sim}$ & $\begin{array}{l}n \\
\hat{n} \\
i \\
i\end{array}$ & $\stackrel{\widehat{N}}{i}$ & 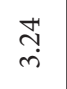 & $\vec{n}$ & $\stackrel{2}{6}$ \\
\hline & ڤ̊ & $\stackrel{\stackrel{?}{i}}{i}$ & $\vec{b}$ & $\stackrel{\text { ㄱ. }}{\wedge}$ & $\begin{array}{l}\tilde{N} \\
0\end{array}$ & $\stackrel{N}{\curvearrowright}$ & $\stackrel{\stackrel{L}{m}}{m}$ & $\begin{array}{l}\text { \& } \\
\text { đi }\end{array}$ & $\underset{ন}{\stackrel{J}{n}}$ & $\stackrel{\text { I̊ }}{0}$ & $\stackrel{\stackrel{ }{i}}{\stackrel{i}{i}}$ & $\begin{array}{l}0 \\
\stackrel{\leftrightarrow}{+} \\
0\end{array}$ & 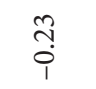 & $\stackrel{\widetilde{\vartheta}}{i}$ & $\stackrel{N}{\stackrel{N}{n}}$ & $\stackrel{\infty}{\stackrel{\infty}{i}}$ & f્ \\
\hline & $\begin{array}{l}\infty \\
\infty \\
\infty \\
\infty\end{array}$ & $\begin{array}{l}\text { oे } \\
\text { in }\end{array}$ & $\begin{array}{l}\text { L } \\
\text { on } \\
\text { in }\end{array}$ & $\begin{array}{l}\stackrel{+}{0} \\
\stackrel{\infty}{-}\end{array}$ & 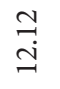 & $\stackrel{\stackrel{\sim}{+}}{+}$ & 0 & 0 & $\stackrel{m}{m}$ & $\stackrel{\sharp !}{0}$ & $\begin{array}{l}\mathcal{N} \\
\stackrel{i}{i}\end{array}$ & 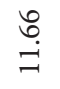 & $\stackrel{\infty}{\circ}$ & $\stackrel{\text { fof }}{\rightarrow}$ & $\begin{array}{l}\stackrel{L}{n} \\
\stackrel{i n}{n}\end{array}$ & $\stackrel{\leftrightarrow}{\stackrel{+}{+}}$ & in \\
\hline & 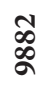 & $\stackrel{\infty}{-}$ & $\stackrel{\Re}{\stackrel{\sim}{+}}$ & 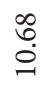 & $\begin{array}{l}\text { Oे } \\
\text { in }\end{array}$ & $\begin{array}{l}\stackrel{2}{0} \\
\stackrel{\leftrightarrow}{\oplus}\end{array}$ & $\stackrel{\hat{m}}{m}$ & 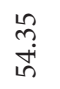 & $\overrightarrow{6}$ & 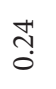 & 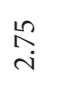 & $\begin{array}{l}\dot{0} \\
\stackrel{2}{2}\end{array}$ & $\stackrel{f}{\stackrel{f}{+}}$ & $\stackrel{\infty}{\vec{i}}$ & $\underset{\text { సे }}{\text { ते }}$ & $\vec{n}$ & $\stackrel{\stackrel{m}{?}}{\stackrel{n}{n}}$ \\
\hline \multirow{2}{*}{ 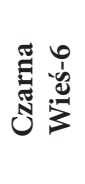 } & $\begin{array}{l}\infty \\
\stackrel{0}{N} \\
\end{array}$ & $\stackrel{\infty}{\stackrel{\infty}{\infty}}$ & $\stackrel{\widehat{H}}{+}$ & 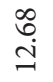 & $\begin{array}{l}\stackrel{L}{\infty} \\
\stackrel{\infty}{\triangle}\end{array}$ & $\stackrel{f}{\stackrel{0}{=}}$ & $\stackrel{\Delta}{\circ}$ & $\begin{array}{l}\tilde{\sigma} \\
\vec{\forall}\end{array}$ & 点 & $\stackrel{\sharp}{0}$ & $\begin{array}{l}\stackrel{゚}{i} \\
\text { in }\end{array}$ & $\begin{array}{l}\stackrel{\widehat{\lambda}}{\mathrm{N}} \\
\text { }\end{array}$ & $\begin{array}{l}\infty \\
\infty \\
0 \\
0\end{array}$ & ભે & $\stackrel{\text { హे }}{+}$ & $\stackrel{\stackrel{\leftrightarrow}{n}}{n}$ & $\underset{+}{\stackrel{H}{+}}$ \\
\hline & ্ֻণ & $\stackrel{m}{\stackrel{m}{n}}$ & $\begin{array}{l}\tilde{O} \\
\varrho\end{array}$ & $\frac{\partial}{\vec{m}}$ & $\begin{array}{l}\mathscr{L} \\
\infty \\
\infty \\
m\end{array}$ & $\stackrel{\vec{\jmath}}{\exists}$ & 0 & 0 & $\stackrel{\widehat{\hat{i}}}{ }$ & $\stackrel{\text { ft }}{0}$ & $\underset{\mathrm{N}}{\mathbb{N}}$ & $\begin{array}{l}\stackrel{10}{0} \\
\stackrel{\infty}{\rightarrow}\end{array}$ & à & $\stackrel{\vec{i}}{\vec{i}}$ & $\begin{array}{l}\text { ff } \\
\text { in }\end{array}$ & $\overrightarrow{\vec{r}}$ & $\stackrel{n}{\rightarrow}$ \\
\hline \multirow{2}{*}{ 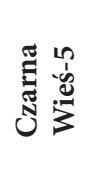 } & 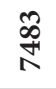 & $\begin{array}{l}\hat{\widehat{\lambda}} \\
\hat{\imath}\end{array}$ & $\stackrel{\sqrt[n]{?}}{\stackrel{-}{a}}$ & $\begin{array}{l}\stackrel{H}{*} \\
\underset{\sim}{n}\end{array}$ & $\underset{+}{\stackrel{\alpha}{d}}$ & గñ & 0 & 0 & $\stackrel{\infty}{\overbrace{0}^{\infty}}$ & $\stackrel{m}{-}$ & f્ & $\stackrel{\stackrel{2}{二}}{\underset{+}{-}}$ & $\stackrel{0}{\stackrel{0}{0}}$ & $N$ & ָ̧ & 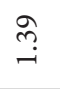 & ชี \\
\hline & 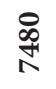 & $\begin{array}{l}\stackrel{\mathfrak{n}}{\mathrm{I}} \\
\mathrm{I}\end{array}$ & స్ & $\stackrel{\stackrel{n}{h}}{=}$ & $\stackrel{\Delta}{N}$ & $\begin{array}{l}\stackrel{\circ}{+} \\
\stackrel{\sim}{N}\end{array}$ & $\begin{array}{l}\tilde{\infty} \\
\underset{\sim}{\tilde{j}}\end{array}$ & 0 & $\vec{త}$ & $\stackrel{\leftrightarrow}{m}$ & $\begin{array}{l}\stackrel{L}{h} \\
\text { in }\end{array}$ & $\vec{\sim}$ & & 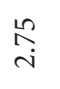 & ir & $\stackrel{\text { oे }}{\text { pr }}$ & $\begin{array}{l}\text { D } \\
\text { i }\end{array}$ \\
\hline \multirow{3}{*}{ 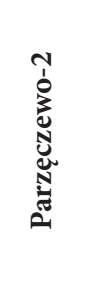 } & 享 & $\begin{array}{l}\hat{\leftrightarrow} \\
\stackrel{+}{+}\end{array}$ & 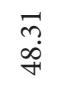 & 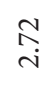 & $\stackrel{m}{-}$ & 0 & 0 & 0 & ก̊ & $\begin{array}{l}\stackrel{L}{L} \\
0\end{array}$ & $\begin{array}{l}\stackrel{\sim}{n} \\
\text { in }\end{array}$ & \begin{tabular}{l}
\multirow{2}{*}{} \\
$\stackrel{-}{\circ}$
\end{tabular} & $\stackrel{\infty}{\circ}$ & ذْتْ & $\ddot{b}$ & $\begin{array}{l}\stackrel{0}{0} \\
\hat{0}\end{array}$ & $\stackrel{\infty}{\circ}$ \\
\hline & ڤ్రి & $\begin{array}{l}l \\
\swarrow \\
\infty \\
\infty\end{array}$ & बे & $\begin{array}{l}\tilde{A} \\
\stackrel{\omega}{n}\end{array}$ & $\underset{\sim}{\tilde{C}}$ & 0 & 0 & 0 & $\stackrel{8}{\circ}$ & $\stackrel{2}{\rightleftarrows}$ & $\stackrel{\infty}{\stackrel{\infty}{-}}$ & $\stackrel{\text { }}{\stackrel{\text { F }}{ت}}$ & $\hat{o}$ & m & $\vec{\sigma}$ & กิ & $\underset{0}{\stackrel{H}{0}}$ \\
\hline & ڤิ & $\stackrel{\hat{m}}{\stackrel{\Theta}{-}}$ & $\begin{array}{l}\stackrel{0}{0} \\
\stackrel{0}{0}\end{array}$ & 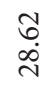 & 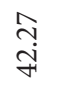 & $\hat{\sigma}$ & 0 & 0 & $\stackrel{\sim}{\stackrel{n}{i}}$ & $\underset{0}{\overrightarrow{0}}$ & 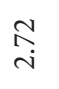 & $\stackrel{\text { Ln }}{\stackrel{2}{S}}$ & $\tilde{o}$ & $\stackrel{H}{i}$ & $\stackrel{\substack{n \\
\infty \\
\infty}}{0}$ & $\underset{\text { iे }}{\text { iे }}$ & 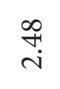 \\
\hline \multirow{3}{*}{ 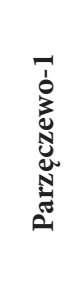 } & ల్రి & $\stackrel{\partial}{\dot{m}}$ & $\stackrel{\Re}{\stackrel{n}{n}}$ & aे & $\begin{array}{l}\text { ¿ें } \\
\text { ते }\end{array}$ & $\begin{array}{l}\hat{y} \\
\text { in }\end{array}$ & 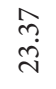 & 0 & $\stackrel{\hat{f}}{+}$ & $\vec{m}$ & 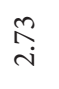 & ָे & $\stackrel{\infty}{0}$ & $\underset{i}{\stackrel{H}{i}}$ & $\begin{array}{l}\infty \\
0 \\
0\end{array}$ & $\overrightarrow{\widetilde{r}}$ & $\exists$ \\
\hline & $\overline{\widehat{B}}$ & $\begin{array}{l}\hat{\infty} \\
\stackrel{i}{i}\end{array}$ & $\stackrel{\infty}{\underset{m}{n}}$ & $\stackrel{\vec{N}}{N}$ & $\begin{array}{l}\vec{\sigma} \\
\stackrel{\leftrightarrow}{\omega}\end{array}$ & $\begin{array}{l}\vec{m} \\
\stackrel{\infty}{\rightarrow}\end{array}$ & 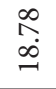 & in & $\stackrel{\infty}{\stackrel{\infty}{+}}$ & $\hat{\overbrace{}}$ & $\begin{array}{l}\stackrel{8}{0} \\
i\end{array}$ & $\underset{\sim}{\stackrel{\Delta}{\sim}}$ & $\stackrel{\tilde{b}}{0}$ & $\stackrel{\infty}{\stackrel{\infty}{\sim}}$ & గ̂nู & $\underset{\sim}{\stackrel{\sim}{+}}$ & 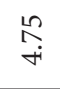 \\
\hline & గ్రి & $\stackrel{\stackrel{n}{\sim}}{\underset{I}{ت}}$ & $\begin{array}{l}0 \\
0 \\
0\end{array}$ & శี & $\begin{array}{l}\hat{\alpha} \\
\infty \\
\text { ల) }\end{array}$ & $\begin{array}{l}\mathscr{L} \\
\infty \\
\infty\end{array}$ & 0 & 0 & $\stackrel{\widetilde{N}}{\rightarrow}$ & $\begin{array}{l}\stackrel{\bullet}{0} \\
:\end{array}$ & $\begin{array}{l}\qquad 0 \\
i \\
i\end{array}$ & 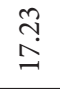 & $\tilde{\sigma}$ & $\stackrel{\vec{i}}{\mathrm{i}}$ & $\begin{array}{l}\infty \\
\infty \\
i \\
i\end{array}$ & $\stackrel{\sim}{i}$ & $\overrightarrow{\widetilde{N}}$ \\
\hline & & \multicolumn{13}{|c|}{ Lつ-0.J!!W } & \multicolumn{3}{|c|}{ [\%] צWN } \\
\hline & & \multicolumn{8}{|c|}{$\overline{2}$} & \multirow[b]{2}{*}{ 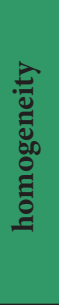 } & \multirow{2}{*}{ 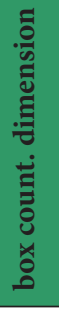 } & \multirow{2}{*}{ 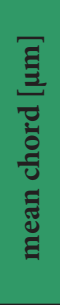 } & \multirow{2}{*}{ 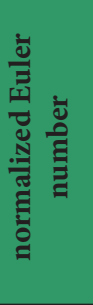 } & \multirow{2}{*}{ 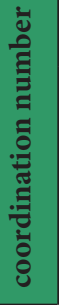 } & & \multirow[b]{2}{*}{ है } & \multirow[b]{2}{*}{$\stackrel{0}{\underline{z}}$} \\
\hline & 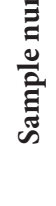 & - & $\exists$ & $\Xi$ & $\geq$ & $>$ & 5 & $\overline{7}$ & 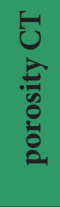 & & & & & & & & \\
\hline & & - & $N$ & $n$ & 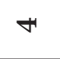 & in & 6 & N & $\infty$ & $a$ & 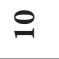 & $z$ & $\simeq$ & 2 & $\Xi$ & 12 & $\stackrel{0}{=}$ \\
\hline
\end{tabular}




\begin{tabular}{|c|c|c|c|c|c|c|c|c|c|c|c|c|c|c|c|}
\hline \multirow{9}{*}{ 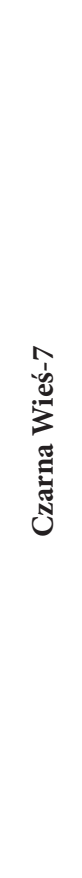 } & ڤิ & $\stackrel{m}{\longrightarrow}$ & $\underset{\infty}{\vec{\infty}}$ & $\begin{array}{l}\text { ते } \\
\text { ஸे }\end{array}$ & $\stackrel{m}{+}$ & $\stackrel{\text { Ln }}{+}$ & $\stackrel{n}{\circ}$ & $\begin{array}{l}\stackrel{m}{m} \\
\stackrel{+}{+}\end{array}$ & $\stackrel{20}{0}$ & $\stackrel{\Re}{\hat{0}}$ & $\begin{array}{l}\infty \\
\stackrel{\infty}{n} \\
0\end{array}$ & $\stackrel{ت}{\dot{I}}$ & $\stackrel{\vec{d}}{\dot{d}}$ & تُ & 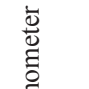 \\
\hline & ฉ̊ & $\stackrel{N}{\stackrel{N}{+}}$ & $\begin{array}{l}\vec{\infty} \\
\stackrel{0}{\oplus}\end{array}$ & 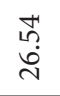 & ڤે & $\stackrel{\sim}{\underset{f}{f}}$ & $\stackrel{\text { Эે }}{=}$ & $\stackrel{0}{\sim}$ & in & $\begin{array}{l}\not \\
\vdots \\
0\end{array}$ & $\stackrel{\infty}{\dddot{0}}$ & 足 & in & $\stackrel{\Re}{i}$ & $\begin{array}{l}\text { 商 } \\
\text { 严 }\end{array}$ \\
\hline & ڤ̆ & $\begin{array}{l}\stackrel{\leftrightarrow}{G} \\
=\end{array}$ & $\stackrel{\infty}{\infty}$ & 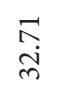 & $\underset{\mathrm{J}}{\mathfrak{J}}$ & $\tilde{m}$ & $\underset{\infty}{\mathbb{N}}$ & $\stackrel{\nearrow}{ت}$ & \begin{tabular}{l}
$?$ \\
\hdashline \\
0
\end{tabular} & $\stackrel{0}{\circ}$ & $\ddot{0}$ & $\stackrel{\check{j}}{\dot{j}}$ & تُ & تُ & 1 \\
\hline & ळ̊ & $\stackrel{\stackrel{2}{+}}{\stackrel{0}{0}}$ & $\vec{n}$ & $\begin{array}{l}\dot{D} \\
\dot{0}\end{array}$ & $\stackrel{0}{\stackrel{\oplus}{\oplus}}$ & $\underset{\infty}{\tilde{\infty}}$ & $\stackrel{n}{\varrho}$ & $\stackrel{\infty}{\stackrel{\leftrightarrow}{0}}$ & กิ & $\stackrel{\infty}{\circ}$ & $\stackrel{m}{\circ}$ & ت્ટ & تُ & $\stackrel{0}{-}$ & $\stackrel{\circ}{\circ}$ \\
\hline & ڤ̊ & $\begin{array}{l}\hat{\widehat{A}} \\
\text { I }\end{array}$ & $\underset{\infty}{\mathbb{Z}}$ & $\begin{array}{l}\stackrel{\sigma}{0} \\
\stackrel{m}{\oplus}\end{array}$ & $\hat{\mathrm{I}}$ & $\underset{+}{\stackrel{6}{+}}$ & $\begin{array}{l}\text { ப் } \\
\stackrel{0}{0}\end{array}$ & $\stackrel{m}{m}$ & ণิ & $\stackrel{+}{\stackrel{H}{0}}$ & $\stackrel{\circ}{0}$ & $\stackrel{\infty}{\stackrel{\infty}{i}}$ & $g$ & 酋 & 范 \\
\hline & बूर & $\begin{array}{l}\vec{a} \\
\stackrel{\mathrm{J}}{ }\end{array}$ & $\begin{array}{l}\hat{\sigma} \\
\stackrel{0}{\circ}\end{array}$ & $\stackrel{7}{\stackrel{2}{n}}$ & $\stackrel{\sim}{\rightarrow}$ & $\begin{array}{l}\stackrel{n}{0} \\
\stackrel{n}{n}\end{array}$ & $\stackrel{F}{\exists}$ & $\vec{\sigma}$ & $\stackrel{\stackrel{\sim}{?}}{0}$ & $\stackrel{\infty}{\stackrel{\infty}{0}}$ & ชุ & ت્ય & $\stackrel{ت}{\dot{d}}$ & $\begin{array}{l}\infty \\
\text { i }\end{array}$ & च \\
\hline & ڤ̊ & 人ે & $\begin{array}{l}\stackrel{L}{n} \\
\stackrel{i n}{n}\end{array}$ & $\stackrel{\stackrel{P}{F}}{\vec{F}}$ & ને & $\hat{n}$ & $\stackrel{\mathscr{L}}{=}$ & $\overbrace{0}^{\infty}$ & $\stackrel{1}{0}$ & $\stackrel{+}{\stackrel{H}{0}}$ & $\stackrel{\infty}{\stackrel{0}{0}}$ & ભે & ت্] & $\stackrel{?}{\stackrel{f}{-}}$ & \\
\hline & $\begin{array}{l}\infty \\
\infty \\
\infty \\
a\end{array}$ & $\stackrel{9}{=}$ & $\begin{array}{l}\qquad 0 \\
0\end{array}$ & $\vec{F}$ & $\stackrel{n}{=}$ & $\stackrel{\infty}{-\infty}$ & $\stackrel{\leftrightarrow}{+}$ & $\overrightarrow{\widetilde{T}}$ & $\stackrel{\infty}{\stackrel{0}{0}}$ & ஸे & $\stackrel{\vec{b}}{-}$ & تُ & ت] & تُ & \\
\hline & $\begin{array}{l}\text { مू } \\
\approx \\
\approx\end{array}$ & $\begin{array}{l}\stackrel{0}{0} \\
\stackrel{m}{-}\end{array}$ & 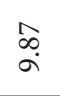 & $\begin{array}{l}\text { ơ } \\
\text { L्र }\end{array}$ & 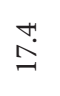 & $\widehat{త}$ & فُذْ & ف묘. & نُّ & ذّ & $\dot{\dot{D}}$ & 莺 & $\underset{\text { I }}{\text { i }}$ & 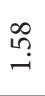 & \\
\hline 胥 & $\stackrel{\infty}{\stackrel{0}{0}}$ & 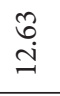 & $\stackrel{a}{\circ}$ & $\vec{a}$ & $\begin{array}{l}\infty \\
\stackrel{0}{n} \\
\varrho\end{array}$ & $\begin{array}{l}\infty \\
\infty \\
\infty \\
\infty \\
\infty\end{array}$ & $\stackrel{\bullet}{\stackrel{\bullet}{\Xi}}$ & $\stackrel{\leftrightarrow}{\Omega}$ & $\stackrel{\infty}{!}$ & $\stackrel{\substack{n \\
0}}{\hat{0}}$ & $\stackrel{0}{0}$ & تُ & $\stackrel{ت}{\dot{d}}$ & تُ & \\
\hline 3. & 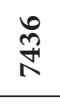 & $\stackrel{\mathscr{\imath}}{\stackrel{\Xi}{=}}$ & $\begin{array}{l}\vec{\infty} \\
\dot{i n}\end{array}$ & 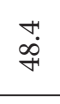 & $\stackrel{2}{\stackrel{2}{\circ}}$ & $\begin{array}{l}\mathscr{L} \\
\stackrel{\infty}{0} \\
0\end{array}$ & $a$ & $\stackrel{\leftrightarrow}{m}$ & 3 & $\stackrel{0}{0}$ & $\stackrel{7}{0}$ & & $\stackrel{\infty}{\stackrel{\infty}{\rightarrow}}$ & $\stackrel{\infty}{-}$ & \\
\hline : & $\stackrel{\infty}{\stackrel{\infty}{N}}$ & $\vec{\sigma}$ & ڤે̣ & $\stackrel{\infty}{\stackrel{\infty}{\wedge}}$ & $\stackrel{\vec{i}}{\text { in }}$ & 菅 & $\begin{array}{l}\stackrel{0}{m} \\
\dot{m}\end{array}$ & 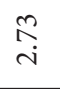 & $\stackrel{0}{\circ}$ & $\hat{o}$ & กั & تُ & $\stackrel{\check{g}}{\mathrm{~g}}$ & تُ & $r^{\circ}$ \\
\hline $\mathrm{O}$ & $\begin{array}{l}\infty \\
\stackrel{\infty}{N} \\
\end{array}$ & 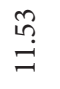 & $\begin{array}{l}\text { مे } \\
\text { in }\end{array}$ & $\begin{array}{l}\hat{i n} \\
\text { in } \\
\infty \\
\alpha\end{array}$ & $\underset{\infty}{\vec{\infty}}$ & L̊ & $\stackrel{\infty}{\stackrel{\text { in }}{0}}$ & $\stackrel{\vec{\sigma}}{-}$ & $\stackrel{0}{0}$ & in & $\stackrel{\sigma}{0}$ & ت্] & تُ & تُ & \\
\hline $\mathcal{I}$ & ఫ్ & 年 & $\begin{array}{l}\vec{\infty} \\
0 \\
0\end{array}$ & 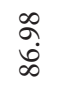 & $\begin{array}{l}\infty \\
\infty \\
\text { r. }\end{array}$ & $\stackrel{\text { L̊ }}{0}$ & $\stackrel{n}{\longrightarrow m}$ & $\stackrel{\text { J }}{\text {. }}$ & 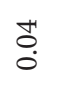 & घn? & $\stackrel{\widetilde{r}}{0}$ & تُ & $\stackrel{\overbrace{}}{\dot{g}}$ & تُ & \\
\hline 胥 & ڤ్రి & in & $\stackrel{\bullet}{\circ}$ & $\begin{array}{l}\qquad 0 \\
\infty \\
\infty \\
\infty\end{array}$ & $\stackrel{\infty}{\infty}$ & $\stackrel{2}{\circ}$ & $\stackrel{\infty}{\stackrel{\infty}{m}}$ & $\stackrel{\overbrace{}}{\rightrightarrows}$ & $\stackrel{\Delta}{0}$ & $\stackrel{m}{3}$ & f̊: & ت্] & ت্] & تُ & \\
\hline$\Xi$ & గ్రీ & 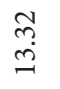 & $\stackrel{\hat{\sigma}}{+}$ & $\begin{array}{l}\hat{b} \\
\text { i }\end{array}$ & $\stackrel{+}{=}$ & $\stackrel{\overbrace{}}{\stackrel{\overbrace{}}{-}}$ & $\stackrel{20}{a}$ & $\stackrel{\text { ? }}{-}$ & $\stackrel{8}{\circ}$ & ?. & $\stackrel{\stackrel{\sim}{T}}{0}$ & ت্] & ت্] & تُ & \\
\hline & గ్రి & $\stackrel{\ddot{\sim}}{\stackrel{\longrightarrow}{\sim}}$ & $\tilde{N}$ & $\begin{array}{l}\hat{m} \\
\dot{f}\end{array}$ & $\stackrel{9}{=}$ & $\stackrel{m}{=}$ & 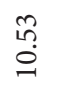 & $\vec{S}$ & oे & 苞 & $\stackrel{m}{0}$ & تُ & ت्] & $\stackrel{\text { }}{\rightarrow}$ & \\
\hline 胥 & $\bar{B}$ & $\begin{array}{l}\vec{n} \\
\underline{n}\end{array}$ & $\begin{array}{l}\infty \\
\infty \\
\infty\end{array}$ & $\overrightarrow{\mathcal{H}}$ & ֶี & $\stackrel{\sim}{\sim}$ & 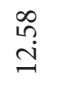 & $\stackrel{\hat{m}}{\stackrel{+}{+}}$ & 7 & in & $\vec{\Im}$ & 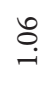 & تृ & $\stackrel{m}{=}$ & \\
\hline$\approx$ & ల్లి & $\stackrel{H}{3}$ & $\underset{\forall}{F}$ & $\begin{array}{l}\text { âे } \\
\text { in }\end{array}$ & $\underset{\infty}{\overrightarrow{0}}$ & તิ่ & જิ & $\stackrel{\widetilde{N}}{\rightarrow}$ & $\stackrel{0}{\circ}$ & $\stackrel{\infty}{\stackrel{\infty}{0}}$ & $\vec{\jmath}$ & تُ & ت্] & $\stackrel{\check{I}}{\longrightarrow}$ & \\
\hline & & & ] ४и & & ¿ & $\overline{\mathrm{g}}$ & & & & & 1 & & & & \\
\hline & 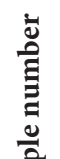 & $\begin{array}{l}\stackrel{3}{0} \\
\frac{0}{0} \\
\vdots\end{array}$ & $\begin{array}{l}\stackrel{3}{0} \\
0 \\
\vdots \\
\vdots \\
0\end{array}$ & $\begin{array}{l}\overline{0} \\
\frac{0}{3} \\
\frac{0}{0} \\
\overline{0}\end{array}$ & $\begin{array}{l}\hat{\bar{n}} \\
\overline{0} \\
\stackrel{0}{0} \\
\stackrel{0}{2}\end{array}$ & 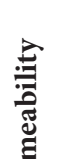 & 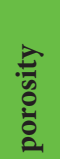 & $\begin{array}{l}\underline{\Xi} \\
\dot{\Xi} \\
\hat{\vdots}\end{array}$ & $\begin{array}{l}\text { है } \\
\text { 离 } \\
\text { है }\end{array}$ & $\begin{array}{l}\underline{\mathrm{E}} \\
\overrightarrow{\hat{A}}\end{array}$ & 产 & & مِّ & & \\
\hline & ڤ్ & $\stackrel{0}{9}$ & ஜँ๊ &. & $\bar{I}$ & 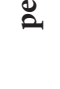 & $\bar{I}$ & $\stackrel{200}{I}$ & ๕̆ & $\stackrel{2}{2}$ & 完 & $\star$ & $\lambda$ & $\mathbf{N}$ & \\
\hline & & $\bumpeq$ & $\stackrel{\infty}{\sim}$ & 2 & సి & $\overline{\boldsymbol{N}}$ & $\tilde{N}$ & $\tilde{\sim}$ & $\underset{N}{\mathbb{N}}$ & $\stackrel{\text { L }}{4}$ & i & $\hat{\imath}$ & $\stackrel{\infty}{\text { ஸे }}$ & సి & \\
\hline
\end{tabular}




\begin{tabular}{|c|c|c|c|c|c|c|c|c|c|c|c|c|c|c|c|c|c|}
\hline \multirow{7}{*}{ 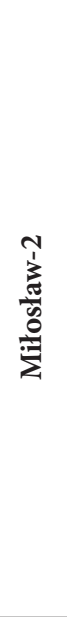 } & $\begin{array}{c}\text { సิ } \\
\text { בิ }\end{array}$ & రి & $\begin{array}{l}\vec{b} \\
\ddot{+}\end{array}$ & $\stackrel{\stackrel{m}{+}}{\sim}$ & $\stackrel{\overbrace{}}{\stackrel{2}{\Delta}}$ & ָે & જે & 0 & $\stackrel{\tilde{m}}{\tilde{m}}$ & $\hat{\tilde{o}}$ & $\begin{array}{l}\overrightarrow{0} \\
i\end{array}$ & $\stackrel{\overbrace{}}{\mathrm{I}}$ & f̊ & $\stackrel{\circ}{\stackrel{-}{-}}$ & $\vec{i}$ & $\stackrel{\overbrace{}}{+}$ & 茴 \\
\hline & बิ & $\stackrel{\Omega}{\infty}$ & $\begin{array}{l}n \\
\infty \\
\infty \\
\end{array}$ & $\vec{\sim}$ & ते & 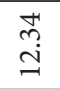 & 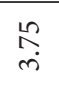 & 0 & $\stackrel{\Re}{\stackrel{\sim}{+}}$ & ֶֻ & $\begin{array}{l}\infty \\
\stackrel{\infty}{i}\end{array}$ & $\begin{array}{l}\vec{n} \\
\stackrel{n}{n}\end{array}$ & $\stackrel{\text { ff }}{0}$ & 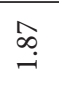 & $\underset{m}{F}$ & $\begin{array}{l}\infty \\
\infty \\
i n\end{array}$ & 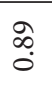 \\
\hline & 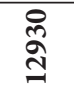 & $\vec{\sigma}$ & $\begin{array}{l}\text { oे } \\
\stackrel{9}{9}\end{array}$ & 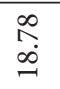 & $\begin{array}{l}\stackrel{\gtrless}{\hat{n}} \\
\text { na }\end{array}$ & $\begin{array}{l}\hat{\infty} \\
\stackrel{0}{0} \\
\stackrel{0}{0}\end{array}$ & ஜ̊ & 0 & $\underset{+}{\tilde{f}}$ & $\tilde{\widetilde{o}}$ & 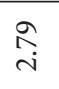 & $\begin{array}{l}\vec{\sigma} \\
\underset{+}{+}\end{array}$ & ஸे & $\stackrel{\substack{\infty \\
\rightarrow}}{-}$ & $\stackrel{?}{m}$ & $\ddot{H}$ & ? \\
\hline & $\begin{array}{l}\text { i⿱ } \\
\text { న్ }\end{array}$ & $\stackrel{\llcorner}{\leftrightarrow}$ & $\underset{+}{\stackrel{a}{+}}$ & $\underset{-}{\vec{\sigma}}$ & $\underset{-}{\sharp}$ & $\tilde{ֶ}$ & $\underset{-}{\sharp}$ & 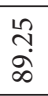 & $\begin{array}{l}\infty \\
\stackrel{0}{\exists} \\
\end{array}$ & $\stackrel{\overrightarrow{0}}{0}$ & $\begin{array}{l}\vec{\infty} \\
i\end{array}$ & $\begin{array}{l}\text { मे } \\
\text { ते }\end{array}$ & $\stackrel{\hat{O}}{i}$ & $\underset{i}{\stackrel{H}{i}}$ & 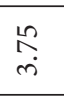 & $\stackrel{\circ}{\circ}$ & 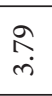 \\
\hline & $\begin{array}{l}\text { ָิ } \\
\text { ָิ }\end{array}$ & $\stackrel{\sim}{\sim}$ & 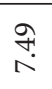 & oे & $\begin{array}{l}\infty \\
\stackrel{\infty}{+} \\
\stackrel{\rightarrow}{7}\end{array}$ & 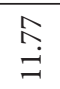 & $\stackrel{\hat{m}}{i}$ & 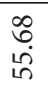 & $\stackrel{\infty}{\stackrel{\infty}{\wedge}}$ & $\stackrel{\infty}{\stackrel{\infty}{0}}$ & $\stackrel{\infty}{\stackrel{\infty}{i}}$ & $\begin{array}{l}+ \\
\infty \\
\stackrel{0}{\sigma}\end{array}$ & 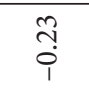 & $\stackrel{\stackrel{m}{r}}{i}$ & $\begin{array}{l}\stackrel{\circ}{\leftrightarrow} \\
i\end{array}$ & 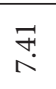 & $\stackrel{\vec{f}}{+}$ \\
\hline & 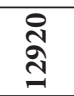 & $\underset{0}{\infty}$ & $\stackrel{m}{\stackrel{2}{a}}$ & $\begin{array}{l}\stackrel{f}{f} \\
\stackrel{\infty}{\rightarrow}\end{array}$ & $\begin{array}{l}\infty \\
\stackrel{2}{ } \\
\infty \\
\infty\end{array}$ & $\begin{array}{l}\infty \\
\stackrel{\infty}{n}\end{array}$ & S. & 0 & ஜூ & $\stackrel{\text { J̦ }}{0}$ & $\stackrel{\infty}{i}$ & $\begin{array}{l}\hat{O} \\
\dot{\omega}\end{array}$ & $\stackrel{\circ}{\circ}$ & $\stackrel{m}{\vec{i}}$ & $\underset{i}{\stackrel{H}{i}}$ & $\underset{6}{ \pm}$ & $\stackrel{\text { If }}{-}$ \\
\hline & $\begin{array}{l}\text { సิ } \\
\text { (n) }\end{array}$ & $\begin{array}{l}\infty \\
\infty \\
\infty\end{array}$ & $\begin{array}{l}\hat{\sigma} \\
\stackrel{0}{0}\end{array}$ & $\stackrel{\hat{n}}{\hat{n}}$ & Fे & $\underset{m}{\vec{m}}$ & $\stackrel{\circ}{\circ}$ & $\underset{\substack{N\\
}}{\mathbb{N}}$ & 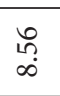 & $\stackrel{\leftrightarrow}{0}$ & $\begin{array}{l}\infty \\
\stackrel{\infty}{i}\end{array}$ & $\stackrel{\sharp}{\leftrightarrows}$ & $\begin{array}{l}\overrightarrow{7} \\
\end{array}$ & $\stackrel{\text { H }}{\mathrm{i}}$ & $\stackrel{f}{-}$ & $\stackrel{\widehat{\widehat{\vartheta}}}{i n}$ & $\underset{\sim}{\stackrel{m}{+}}$ \\
\hline \multirow{6}{*}{ 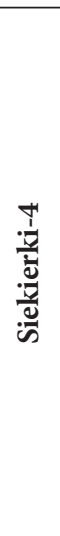 } & $\begin{array}{l}\infty \\
\stackrel{\infty}{\Xi}\end{array}$ & के & $\begin{array}{l}\text { Hे } \\
\stackrel{n}{n}\end{array}$ & $\tilde{\curvearrowright}$ & 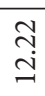 & 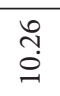 & $\underset{\infty}{\infty}$ & $\underset{\mathrm{N}}{\mathrm{W}}$ & 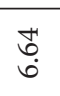 & $\stackrel{m}{0}$ & $\begin{array}{l}\stackrel{+}{\infty} \\
\stackrel{i}{i}\end{array}$ & 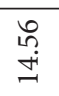 & 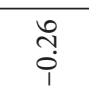 & $\stackrel{\circ}{\stackrel{-}{-}}$ & $\stackrel{\hat{n}}{i}$ & 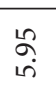 & $\stackrel{\text { Эे }}{\rightarrow}$ \\
\hline & సิ & 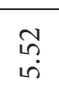 & $\begin{array}{l}\tilde{\beta} \\
\stackrel{+}{-}\end{array}$ & $\begin{array}{l}\text { fे } \\
\stackrel{-}{6}\end{array}$ & $\begin{array}{l}\infty \\
\dot{m}\end{array}$ & $\begin{array}{l}\text { ते } \\
\text { in }\end{array}$ & $\stackrel{\widehat{m}}{\rightarrow}$ & $\begin{array}{l}\Delta \\
\infty\end{array}$ & $\begin{array}{l}\vec{\infty} \\
\text { r. }\end{array}$ & $\stackrel{\text { ֻึ }}{0}$ & $\stackrel{\infty}{\stackrel{\infty}{i}}$ & 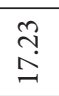 & f̊ & $\underset{\text { సे }}{\text { in }}$ & $\stackrel{\mathscr{n}}{\mathrm{i}}$ & $\begin{array}{l}\stackrel{0}{\infty} \\
\stackrel{+}{+}\end{array}$ & $\stackrel{\text { }}{\stackrel{-}{\rightarrow}}$ \\
\hline & 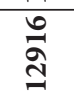 & 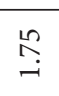 & $\underset{\infty}{\infty}$ & - & $\begin{array}{c}\infty \\
\stackrel{\infty}{0} \\
0\end{array}$ & $\stackrel{\text { L̊ }}{0}$ & 0 & $\begin{array}{l}\infty \\
\stackrel{\alpha}{\alpha}\end{array}$ & $\begin{array}{l}F \\
\stackrel{g}{-}\end{array}$ & $\stackrel{+}{-0}$ & $\begin{array}{c}\widetilde{\alpha} \\
\stackrel{i}{i}\end{array}$ & $\begin{array}{l}\text { nू } \\
\stackrel{\sim}{n}\end{array}$ & $\begin{array}{l}\hat{a} \\
\hat{i}\end{array}$ & $\stackrel{\hat{m}}{i}$ & $\underset{i}{\vec{j}}$ & $\vec{r}$ & 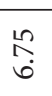 \\
\hline & 㓂 & 号 & $\begin{array}{l}\stackrel{?}{+} \\
\stackrel{+}{+}\end{array}$ & $\begin{array}{l}\infty \\
\stackrel{\text { In }}{\sim}\end{array}$ & $\begin{array}{l}\text { H̊ } \\
\text { îj }\end{array}$ & $\stackrel{\infty}{\stackrel{\infty}{\sharp}}$ & 0 & 0 & $\begin{array}{l}\tilde{b} \\
\text { in }\end{array}$ & $\stackrel{2}{0}$ & $\begin{array}{l}\infty \\
\stackrel{i}{i}\end{array}$ & 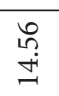 & $\stackrel{0}{0}$ & 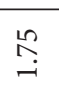 & $\stackrel{\overbrace{}}{i}$ & $\underset{n}{\vec{n}}$ & $\stackrel{\vec{m}}{\dot{m}}$ \\
\hline & $\begin{array}{l}\vec{I} \\
\text { ন }\end{array}$ & $\stackrel{\text { aे }}{\text { Pे }}$ & $\underset{\Omega}{\delta}$ & Әे & H & $\exists$ & $\bar{\sigma}$ & $\begin{array}{l}\infty \\
\infty \\
\dot{0}\end{array}$ & $\hat{\sigma}$ & ָे & $\hat{i}$ & $\begin{array}{l}\underset{J}{*} \\
\stackrel{+}{*}\end{array}$ & $\begin{array}{l}\text { L̊ } \\
\text { in }\end{array}$ & $\underset{-}{\stackrel{\leftrightarrow}{\infty}}$ & $\begin{array}{l}\infty \\
\infty \\
i \\
i\end{array}$ & $\stackrel{\hat{N}}{\text { in }}$ & $\stackrel{\text { ì }}{i}$ \\
\hline & $\frac{m}{\grave{a}}$ & $\wedge$ & $\begin{array}{l}\text { \& } \\
\text { فn }\end{array}$ & $\begin{array}{l}\infty \\
\stackrel{\infty}{n}\end{array}$ & $\begin{array}{l}\infty \\
\stackrel{i}{i}\end{array}$ & $\begin{array}{l}\vec{m} \\
\stackrel{\sim}{*}\end{array}$ & $\begin{array}{l}\stackrel{\infty}{\infty} \\
+\end{array}$ & 0 & $\vec{m}$ & $\stackrel{\vec{ก}}{0}$ & $\begin{array}{l}\stackrel{\leftrightarrow}{\infty} \\
\text { i }\end{array}$ & 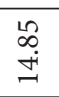 & $\stackrel{0}{0}$ & $\vec{i}$ & $\underset{\mathcal{H}}{\mathrm{I}}$ & $\begin{array}{l}\vec{\infty} \\
\stackrel{\sim}{0}\end{array}$ & î̀ \\
\hline \multirow{8}{*}{$\frac{n}{\frac{1}{20}}$} & ڤે̀ & î & $\begin{array}{l}\infty \\
\stackrel{n}{n} \\
\stackrel{n}{n}\end{array}$ & $\begin{array}{l}\overrightarrow{\vec{\infty}} \\
\dot{\omega}\end{array}$ & 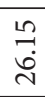 & $\begin{array}{l}\stackrel{0}{1} \\
\text { m. }\end{array}$ & 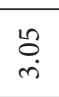 & $\stackrel{\infty}{m_{n}}$ & $\stackrel{\hat{+}}{\hat{+}}$ & त̂ & $\stackrel{\widehat{\overbrace{}}}{\hat{i}}$ & $\begin{array}{l}\infty \\
\infty \\
\\
\end{array}$ & $\begin{array}{l}\overrightarrow{0} \\
0\end{array}$ & $\stackrel{\infty}{\vec{i}}$ & $\underset{m}{m}$ & $\begin{array}{l}\hat{n} \\
\hat{n} \\
i n\end{array}$ & $\vec{\sim}$ \\
\hline & 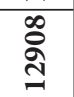 & $\begin{array}{l}\stackrel{n}{n} \\
\stackrel{n}{+}\end{array}$ & $\begin{array}{l}\stackrel{\bullet}{0} \\
=\end{array}$ & $\begin{array}{l}\infty \\
\stackrel{\infty}{\varrho} \\
\stackrel{\sim}{\circ}\end{array}$ & $\begin{array}{l}\hat{\infty} \\
\stackrel{\vec{N}}{+}\end{array}$ & $\begin{array}{l}\stackrel{\Re}{\infty} \\
\stackrel{\infty}{\sim}\end{array}$ & $\begin{array}{l}\tilde{\varrho} \\
\stackrel{-}{-}\end{array}$ & $\exists$ & ?̧ & $\stackrel{\overbrace{}}{0}$ & $\begin{array}{l}\vec{\infty} \\
\stackrel{i}{i}\end{array}$ & $\begin{array}{l}\stackrel{m}{\infty} \\
\stackrel{\infty}{\sim}\end{array}$ & $\stackrel{\leftrightarrow}{0}$ & $\stackrel{\stackrel{\sim}{\sim}}{i}$ & $\begin{array}{l}\infty \\
\stackrel{\sim}{i}\end{array}$ & के & 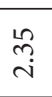 \\
\hline & ڤั้ & $\stackrel{\overbrace{}}{\rightarrow}$ & $\underset{\sim}{\sim}$ & $\hat{\circ}$ & $\vec{J}$ & 0 & 0 & 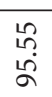 & $\begin{array}{l}\stackrel{\varkappa}{\Im} \\
\underset{+}{\sim}\end{array}$ & $\cong$ & $\begin{array}{l}\hat{\infty} \\
\text { ì }\end{array}$ & ㄱ. & 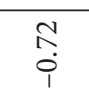 & $\underset{\text { I }}{\mathrm{i}}$ & $\stackrel{m}{m}$ & $\stackrel{?}{\stackrel{P}{+}}$ & $\begin{array}{l}\infty \\
\stackrel{2}{2}\end{array}$ \\
\hline & $\begin{array}{l}\text { पे } \\
\text { ঐิ }\end{array}$ & $\begin{array}{l}\stackrel{a}{0} \\
\text { r. }\end{array}$ & $\underset{\underset{J}{H}}{\stackrel{H}{H}}$ & $\vec{i}$ & 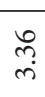 & ầ & $\circ$ & $\underset{\mathbb{N}}{\mathbb{N}}$ & 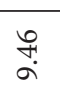 & 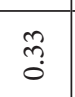 & $\begin{array}{l}\infty \\
\stackrel{i}{i}\end{array}$ & $\begin{array}{l}\underset{\sim}{\mathbb{\sigma}} \\
\text { }\end{array}$ & $\begin{array}{l}\stackrel{2}{n} \\
\stackrel{i}{1}\end{array}$ & $\vec{i}$ & ஸे & mi & $\stackrel{\cong}{\exists}$ \\
\hline & $\begin{array}{l}\text { ڤิ } \\
\text { ָे }\end{array}$ & 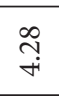 & $\stackrel{\widehat{m}}{\mathrm{i}}$ & $\begin{array}{l}\hat{\beta} \\
\stackrel{+}{+}\end{array}$ & 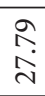 & $\begin{array}{l}\vec{a} \\
\text { ì }\end{array}$ & $\begin{array}{l}\vec{\Xi} \\
\dot{J}\end{array}$ & 0 & 年 & $\stackrel{0}{0}$ & $\stackrel{\infty}{\stackrel{\infty}{i}}$ & 啇 & $\stackrel{+}{0}$ & $\stackrel{\stackrel{\sim}{i}}{\mathrm{i}}$ & $\stackrel{\tilde{r}}{\tilde{r}}$ & 前 & $\stackrel{\sim}{\sim}$ \\
\hline & 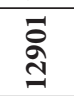 & $=$ & $\begin{array}{l}\text { ? } \\
\text { bे }\end{array}$ & $\begin{array}{l}\stackrel{\hat{n}}{\mathrm{i}} \\
\stackrel{\mathrm{i}}{ }\end{array}$ & 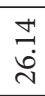 & $\underset{+}{\stackrel{F}{*}}$ & 0 & 0 & $\stackrel{=}{m}$ & $\stackrel{\stackrel{\sim}{\sim}}{0}$ & $\begin{array}{l}\infty \\
\stackrel{i}{i}\end{array}$ & $\begin{array}{l}\tilde{N} \\
\stackrel{\sim}{N}\end{array}$ & 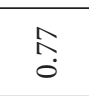 & $\stackrel{\infty}{-}$ & $\hat{i}$ & مे & $\stackrel{?}{\stackrel{f}{+}}$ \\
\hline & $\begin{array}{l}\text { बे } \\
\text { İ }\end{array}$ & $\stackrel{\text { Oे }}{\text {. }}$ & $\begin{array}{l}\overrightarrow{\text { }} \\
\text { ळे }\end{array}$ & $\stackrel{\sim}{\ddot{g}}$ & $\begin{array}{l}\hat{0} \\
\text { ¿े. }\end{array}$ & $\begin{array}{l}\text { ָे } \\
\widetilde{\sim}\end{array}$ & $\overrightarrow{\vec{n}}$ & 0 & $\begin{array}{l}\stackrel{\hat{\Lambda}}{i n} \\
\text { in }\end{array}$ & $\overrightarrow{\widetilde{o}}$ & $\begin{array}{l}\mathscr{\infty} \\
\stackrel{i}{i}\end{array}$ & $\begin{array}{l}\stackrel{2}{2} \\
\underset{-}{+}\end{array}$ & $\begin{array}{l}\stackrel{2}{0} \\
0\end{array}$ & $\stackrel{\hat{a}}{-}$ & $\stackrel{\widehat{i}}{i}$ & $\stackrel{\overbrace{}}{+}$ & $\widetilde{-}$ \\
\hline & 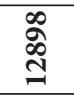 & $\begin{array}{l}\mathscr{8} \\
\stackrel{0}{\circ}\end{array}$ & 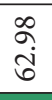 & $\begin{array}{l}\vec{n} \\
\underline{n} \\
\end{array}$ & 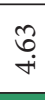 & त̄ & 0 & 0 & $\begin{array}{l}\stackrel{0}{0} \\
\text { in }\end{array}$ & $\stackrel{\sharp}{\stackrel{\Delta}{0}}$ & $\stackrel{\widehat{\lambda}}{i}$ & $\stackrel{\mathbb{N}}{=}$ & ŏ & $\stackrel{\infty}{\stackrel{\infty}{\circ}}$ & $\begin{array}{l}\stackrel{\infty}{\infty} \\
\text { i }\end{array}$ & $\stackrel{H}{\stackrel{H}{n}}$ & 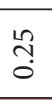 \\
\hline & & \multicolumn{13}{|c|}{ LO-0.J!W } & \multicolumn{3}{|c|}{ [\%] dWN } \\
\hline & & \multicolumn{8}{|c|}{ 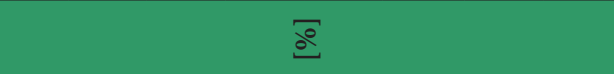 } & \multirow[b]{2}{*}{ 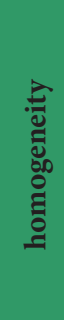 } & \multirow{2}{*}{ 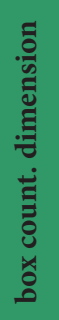 } & \multirow{2}{*}{ 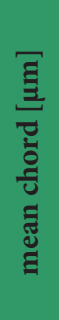 } & \multirow{2}{*}{ 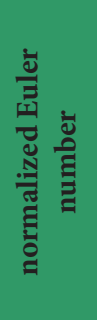 } & \multirow{2}{*}{ 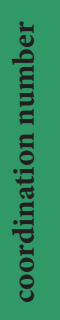 } & \multirow[b]{2}{*}{$\overline{\underline{a}}$} & \multirow[b]{2}{*}{$\underline{\underline{E}}$} & \\
\hline & 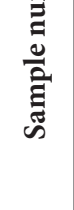 & - & $\exists$ & $\Xi$ & $\geq$ & $>$ & 5 & $\xi$ & $\begin{array}{l}5 \\
\overline{0} \\
\stackrel{\bar{n}}{0} \\
\stackrel{0}{0} \\
\stackrel{0}{0}\end{array}$ & & & & & & & & $\hat{a}$ \\
\hline & & - & $N$ & $n$ & 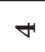 & in & 0 & $n$ & $\infty$ & $a$ & 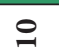 & $z$ & $\simeq$ & $\stackrel{M}{=}$ & $\Xi$ & 12 & 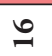 \\
\hline
\end{tabular}




\begin{tabular}{|c|c|c|c|c|c|c|c|c|c|c|c|c|c|c|}
\hline \multirow{7}{*}{ 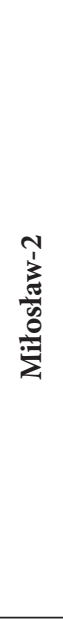 } & స్ & $\wedge$ & 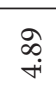 & 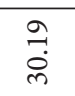 & $\stackrel{\curvearrowright}{\curvearrowright}$ & $\stackrel{0}{0}$ & $\stackrel{m}{\stackrel{n}{n}}$ & $\stackrel{\text { fq }}{\rightarrow}$ & $\stackrel{0}{0}$ & $\tilde{\widetilde{o}}$ & $\stackrel{L}{0}$ & تُ & تُ & تُ \\
\hline & $\overrightarrow{\text { సิ }}$ & $\stackrel{\sim}{\dddot{0}}$ & $\underset{6}{\mathbb{6}}$ & $\begin{array}{l}\stackrel{\circ}{0} \\
\dot{m}\end{array}$ & $\begin{array}{l}\stackrel{2}{\hat{n}} \\
\stackrel{0}{=}\end{array}$ & $\stackrel{2}{0}$ & $\stackrel{7}{\circ}$ & $\stackrel{\mathscr{L}}{\stackrel{2}{\longrightarrow}}$ & $\stackrel{0}{0}$ & $\stackrel{a}{0}$ & $\stackrel{+}{0}$ & $\stackrel{\vec{G}}{\vec{I}}$ & $\stackrel{\check{a}}{\dot{d}}$ & $\stackrel{d}{\dot{I}}$ \\
\hline & $\begin{array}{l}\text { Oิ } \\
\text { సิ }\end{array}$ & $\stackrel{9}{\circ}$ & $\hat{\sigma}$ & $\begin{array}{l}\stackrel{2}{ } \\
\infty \\
\stackrel{m}{m}\end{array}$ & $\stackrel{\widehat{N}}{\Xi}$ & $\stackrel{\infty}{0}$ & $\stackrel{\stackrel{J}{\Xi}}{\Xi}$ & $\stackrel{?}{\rightarrow}$ & $\overrightarrow{0}$ & $\stackrel{n}{\stackrel{0}{0}}$ & 0 & ت্] & $\stackrel{\vec{G}}{\vec{I}}$ & تُ \\
\hline & $\begin{array}{l}\infty \\
\text { స్ }\end{array}$ & 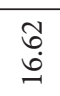 & $\begin{array}{l}\mathbf{D} \\
\stackrel{0}{త} \\
\text { In }\end{array}$ & $\begin{array}{l}\stackrel{0}{\mathrm{i}} \\
\text { in }\end{array}$ & $\hat{\sigma}$ & ڤે & $\begin{array}{l}\stackrel{\infty}{\infty} \\
\stackrel{-}{\triangle}\end{array}$ & $\stackrel{\infty}{\vec{i}}$ & $\overrightarrow{\widetilde{o}}$ & $\stackrel{\text { f }}{\circ}$ & $\stackrel{0}{0}$ & $\stackrel{m}{\longrightarrow}$ & $\stackrel{\stackrel{H}{I}}{\longrightarrow}$ & సે \\
\hline & స్ & $\begin{array}{l}\stackrel{\infty}{\circ} \\
\stackrel{\sim}{\rightarrow}\end{array}$ & $\stackrel{f}{\exists}$ & $\begin{array}{l}\vec{m} \\
\infty \\
\infty\end{array}$ & 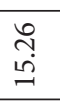 & in & 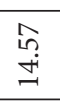 & 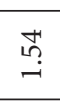 & ָָ & $\stackrel{\text { Ln? }}{0}$ & $\stackrel{\text { L̊ }}{0}$ & $\begin{array}{l}a \\
\text { i }\end{array}$ & $\stackrel{\Re}{-}$ & $\stackrel{\widehat{a}}{-}$ \\
\hline & $\begin{array}{l}\text { ปิ } \\
\text { ปิ }\end{array}$ & 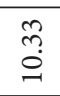 & in? & 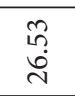 & $\begin{array}{l}\stackrel{2}{\Omega} \\
\stackrel{0}{0}\end{array}$ & $\overrightarrow{0}$ & $\begin{array}{l}\stackrel{\leftrightarrow}{\circ} \\
\stackrel{\leftrightarrow}{\circ}\end{array}$ & $\underset{-}{F}$ & $\stackrel{m}{0}$ & $\stackrel{H}{0}$ & $\stackrel{0}{0}$ & تே & 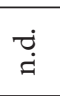 & تُ \\
\hline & సิ & $\stackrel{\text { 点 }}{=}$ & $\stackrel{7}{\circ}$ & 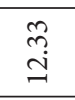 & $\underset{\vec{g}}{\vec{m}}$ & ֻ̊? & 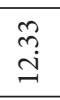 & $\stackrel{\mathscr{h}}{\rightarrow}$ & సิ & 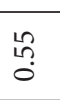 & $\hat{0}$ & $\stackrel{\widetilde{T}}{-}$ & $\stackrel{\widetilde{N}}{-}$ & $\stackrel{\vec{\sigma}}{\vec{\sim}}$ \\
\hline \multirow{6}{*}{ 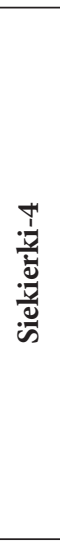 } & 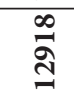 & $\stackrel{\infty}{a}$ & $\stackrel{?}{\stackrel{?}{\sim}}$ & $\begin{array}{l}\underset{+}{+} \\
\underset{\sim}{+}\end{array}$ & $\begin{array}{l}\stackrel{10}{-7} \\
0\end{array}$ & $\stackrel{m}{0}$ & $\ddot{\sigma}$ & 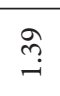 & $\overrightarrow{0}$ & 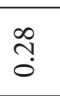 & $\stackrel{\infty}{0}$ & $\stackrel{\vec{C}}{\not}$ & $\stackrel{\infty}{\stackrel{\sim}{\rightarrow}}$ & $\stackrel{\infty}{\stackrel{\infty}{\circ}}$ \\
\hline & 令 & $\underset{\infty}{\partial}$ & 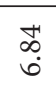 & $\begin{array}{l}\text { ๙े} \\
\tilde{\sim}\end{array}$ & $\vec{n}$ & ğ & $\underset{\infty}{\stackrel{+}{\infty}}$ & $\stackrel{\Re}{\stackrel{\overbrace{}}{-}}$ & $\stackrel{n}{0}$ & 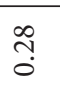 & $\stackrel{0}{0}$ & 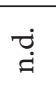 & $\stackrel{\overparen{a}}{\dot{a}}$ & ले \\
\hline & 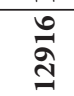 & 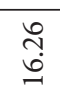 & 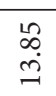 & $\begin{array}{l}\stackrel{\mathscr{O}}{\infty} \\
\stackrel{+}{\sim}\end{array}$ & $\stackrel{\Omega}{\Omega}$ & $\begin{array}{l}\mathscr{L} \\
\infty \\
0 \\
0\end{array}$ & $\begin{array}{l}\tilde{O} \\
\infty \\
\sim\end{array}$ & $\underset{\mathrm{i}}{\stackrel{H}{i}}$ & $\stackrel{\infty}{\stackrel{0}{0}}$ & $\stackrel{0}{\circ}$ & $\stackrel{0}{0}$ & $\underset{\sim}{\stackrel{\sim}{\longrightarrow}}$ & $\stackrel{\widehat{N}}{\rightarrow}$ & $\stackrel{\widehat{\overbrace{}}}{\rightarrow}$ \\
\hline & సิ & भ̊? & $\stackrel{\mathbb{N}}{\mathbb{N}}$ & $\begin{array}{l}\hat{\Lambda} \\
\infty \\
\infty \\
\stackrel{\sim}{d}\end{array}$ & $\begin{array}{l}7 \\
0\end{array}$ & $\stackrel{m}{0}$ & $\begin{array}{l}\hat{A} \\
\stackrel{0}{O}\end{array}$ & $\stackrel{N}{\stackrel{N}{S}}$ & $\stackrel{7}{0}$ & $\stackrel{0}{0}$ & $\stackrel{\circ}{\circ}$ & 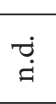 & 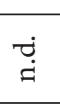 & $\underset{\leftrightarrow}{\dot{I}}$ \\
\hline & ন্ & $\begin{array}{l}\vec{a} \\
\stackrel{\circ}{a}\end{array}$ & $\tilde{o}_{\infty}^{\infty}$ & $\begin{array}{l}\text { के } \\
\text { bे }\end{array}$ & $\begin{array}{l}\tilde{n} \\
\stackrel{\leftrightarrow}{\longrightarrow}\end{array}$ & $\tilde{\widetilde{\sigma}}$ & $\vec{I}$ & $\stackrel{\infty}{-}$ & $\stackrel{7}{0}$ & $\stackrel{0}{\circ}$ & $\stackrel{m}{0}$ & $\stackrel{\text { mे }}{-}$ & $\underset{-}{F}$ & $\stackrel{H}{\longrightarrow}$ \\
\hline & $\frac{m}{\grave{I}}$ & $\begin{array}{l}\infty \\
\infty \\
\infty\end{array}$ & f? & $\begin{array}{c}\hat{n} \\
\stackrel{N}{N}\end{array}$ & aे & $\stackrel{H}{0}$ & $\begin{array}{l}\infty \\
1 \\
0 \\
0\end{array}$ & ڤิ) & $\stackrel{7}{0}$ & $\stackrel{ }{\circ}$ & $\stackrel{0}{\stackrel{0}{0}}$ & تே. & $\stackrel{\vec{g}}{\dot{a}}$ & تُ \\
\hline \multirow{8}{*}{ 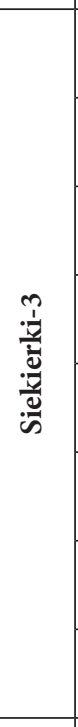 } & 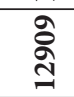 & $\begin{array}{l}\stackrel{8}{0} \\
\stackrel{0}{0}\end{array}$ & $\hat{\hat{\imath}}$ & $\begin{array}{l}\text { నิ } \\
\text { }\end{array}$ & $\begin{array}{l}\stackrel{0}{\circ} \\
\bigcirc\end{array}$ & $\stackrel{\infty}{0}$ & $\vec{\sim}$ & $\stackrel{\sqrt[n]{-}}{-}$ & $\stackrel{\infty}{0}$ & $\stackrel{\infty}{\stackrel{0}{0}}$ & oे. & 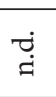 & $\stackrel{\check{g}}{\ddot{d}}$ & $\stackrel{\infty}{-\infty}$ \\
\hline & 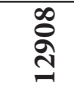 & 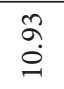 & $\begin{array}{l}\stackrel{\mathscr{T}}{ } \\
\infty \\
\infty\end{array}$ & $\begin{array}{l}\tilde{n} \\
\stackrel{n}{\sim} \\
\stackrel{\sim}{N}\end{array}$ & $\begin{array}{l}\stackrel{H}{\pi} \\
\stackrel{0}{\sim}\end{array}$ & oे. & $\begin{array}{l}\stackrel{\infty}{10} \\
\stackrel{0}{=}\end{array}$ & $\stackrel{\text { ঙે }}{\text { i }}$ & $\overrightarrow{0}$ & $\stackrel{20}{0}$ & $\stackrel{\leftrightarrow}{0}$ & $\stackrel{\leftrightarrow}{\dot{\mu}}$ & $\stackrel{\check{I}}{\dot{I}}$ & $\stackrel{\tilde{i}}{i}$ \\
\hline & 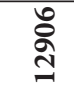 & 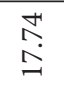 & $\underset{\underset{J}{J}}{F}$ & $\begin{array}{l}\hat{N} \\
\infty \\
\infty\end{array}$ & $\stackrel{\Re}{\stackrel{\Omega}{\Omega}}$ & $\begin{array}{l}\stackrel{\infty}{\stackrel{\infty}{n}} \\
i n\end{array}$ & $\begin{array}{l}\stackrel{\leftrightarrow}{\leftrightarrow} \\
\stackrel{\sim}{\Lambda}\end{array}$ & $\begin{array}{l}\infty \\
\infty \\
\infty\end{array}$ & $\stackrel{H}{-1}$ & 苞 & $\stackrel{\leftrightarrow}{0}$ & $\stackrel{\sim}{-}$ & $\stackrel{\sim}{-}$ & $\stackrel{\sim}{\rightarrow}$ \\
\hline & $\begin{array}{l}\text { ते } \\
\text { త్ }\end{array}$ & 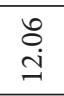 & f̊ & $\stackrel{\stackrel{f}{+}}{\stackrel{\mathrm{i}}{\mathrm{N}}}$ & $\overrightarrow{\mathrm{I}}$ & $\begin{array}{l}\infty \\
0 \\
0\end{array}$ & $\underset{\mathrm{I}}{\mathbb{J}}$ & $\vec{\sigma}$ & $\stackrel{\infty}{\stackrel{0}{0}}$ & ก̂? & $\stackrel{\sigma}{0}$ & $\ddot{d}$ & $\stackrel{\hat{m}}{\rightarrow}$ & $\stackrel{m}{-}$ \\
\hline & ஸे & $\overrightarrow{\mathrm{I}}$ & $\begin{array}{l}\hat{b} \\
\circ\end{array}$ & $\begin{array}{l}\infty \\
\infty \\
\infty \\
\\
\end{array}$ & $\begin{array}{l}\infty \\
\stackrel{\infty}{ \pm} \\
\end{array}$ & $\stackrel{+}{0}$ & $\begin{array}{l}\stackrel{1}{~} \\
\text { İ }\end{array}$ & $\stackrel{\infty}{\stackrel{\infty}{-}}$ & $\stackrel{\infty}{\circ}$ & $\stackrel{\text { In }}{0}$ & $\overrightarrow{0}$ & تே. & تே & ت] \\
\hline & ¿্் & $\ddot{m}$ & ?ु్ & $\vec{\exists}$ & $\hat{0}$ & $\overrightarrow{0}$ & $\begin{array}{l}\text { ఫे } \\
\text { ᄋे }\end{array}$ & $\hat{i}$ & $\stackrel{\infty}{\stackrel{\infty}{0}}$ & $\stackrel{\text { ڤn }}{0}$ & $\stackrel{\text { ô. }}{0}$ & ت્ఝ & ت્ય & $\stackrel{\overbrace{}}{\dot{g}}$ \\
\hline & $\begin{array}{l}\text { बे } \\
\text { తิ }\end{array}$ & $\underset{\infty}{\stackrel{?}{\infty}}$ & $\frac{0}{6}$ & 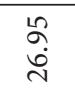 & oे & $=$ & $\vec{m}$ & 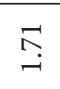 & $\stackrel{7}{7}$ & ֻั & రָ & $\stackrel{\check{g}}{\dot{d}}$ & ت્ય் & $\stackrel{\overbrace{}}{\dot{g}}$ \\
\hline & 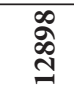 & $\begin{array}{l}\infty \\
\infty \\
0\end{array}$ & & in & S్ర & $\stackrel{\text { ơ }}{0}$ & $\stackrel{\stackrel{\sim}{n}}{\sigma}$ & $\stackrel{m}{m}$ & $\overrightarrow{0}$ & ֻั & $\stackrel{0}{0}$ & $\stackrel{\check{g}}{\dot{d}}$ & $\stackrel{\check{I}}{\dot{I}}$ & تُ \\
\hline & \multirow{4}{*}{ 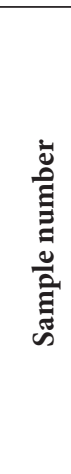 } & \multicolumn{3}{|c|}{ [\%] \&WN } & $\dot{0}$ & $\overline{\mathrm{g}}$ & \multicolumn{4}{|c|}{ s } & 1 & \multirow{2}{*}{\multicolumn{3}{|c|}{$\begin{array}{l}: \\
0 \\
0 \\
0 \\
0 \\
0 \\
0\end{array}$}} \\
\hline & & 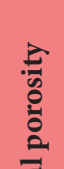 & 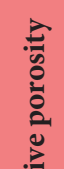 & 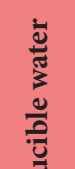 & 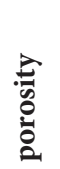 & 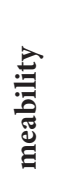 & $\begin{array}{l}\frac{1}{0} \\
0 \\
\dot{0} \\
\stackrel{0}{0}\end{array}$ & $\begin{array}{l}\Xi \\
\vdots \\
\hat{0} \\
\hat{\vdots}\end{array}$ & 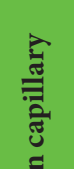 & $\begin{array}{l}\hat{\Xi} \\
\hat{\hat{\theta}} \\
\hat{\mathscr{s}}\end{array}$ & 离离 & & & \\
\hline & & 9 & छँ๊ &.$\Xi$ & 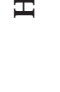 & 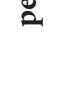 & $\bar{I}$ & $\mathbb{I}^{\infty}$ & § & $a$ & & $x$ & $\lambda$ & $\mathrm{N}$ \\
\hline & & 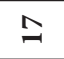 & $\stackrel{\infty}{\sim}$ & 2 & సి & $\overline{\boldsymbol{N}}$ & $\mathcal{N}$ & $\tilde{\sim}$ & $\stackrel{\sim}{N}$ & $\stackrel{2}{\pi}$ & $\stackrel{\text { N }}{ }$ & $\hat{\sim}$ & $\stackrel{\infty}{\sim}$ & సి \\
\hline
\end{tabular}




\begin{tabular}{|c|c|c|c|c|c|c|c|c|c|c|c|c|c|c|c|c|c|}
\hline \multirow{7}{*}{ 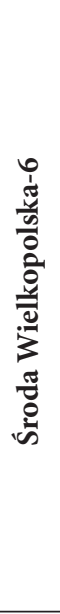 } & స్ & 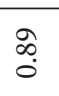 & $\stackrel{\infty}{-\infty}$ & $\stackrel{\text { S̆ }}{0}$ & 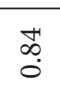 & $\stackrel{\sharp}{0}$ & 0 & $\begin{array}{l}\tilde{N} \\
\text { బू. }\end{array}$ & $\begin{array}{l}\stackrel{\infty}{\infty} \\
\stackrel{\sim}{\sim}\end{array}$ & $\begin{array}{l}0 \\
0\end{array}$ & $\begin{array}{l}\vec{\infty} \\
\vec{i}\end{array}$ & $\begin{array}{l}\infty \\
\stackrel{0}{0} \\
\dot{m}\end{array}$ & $\stackrel{\hat{m}}{\rightarrow}$ & $\underset{\overbrace{}}{\stackrel{\text { I}}{i}}$ & 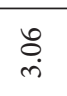 & $\begin{array}{l}\vec{\infty} \\
\dot{n n}\end{array}$ & in \\
\hline & $\underset{\tilde{\sigma}}{\tilde{\sigma}}$ & $\stackrel{\infty}{\stackrel{\infty}{-}}$ & $\begin{array}{l}\tilde{\sigma} \\
\dot{i n}\end{array}$ & $\stackrel{\text { L }}{-}$ & ఫे & $\stackrel{\infty}{\stackrel{0}{0}}$ & గొ & $\begin{array}{l}\text { กิ } \\
\text { \& }\end{array}$ & فِ & त̂̀ & $\hat{i}$ & $\stackrel{m}{\underset{H}{H}}$ & 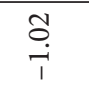 & $\underset{\mathrm{i}}{\overrightarrow{\mathrm{H}}}$ & $\stackrel{m}{i}$ & $\underset{+}{\stackrel{\sigma}{+}}$ & $\begin{array}{l}\vec{H} \\
\stackrel{0}{\circ}\end{array}$ \\
\hline & ๙ิ & 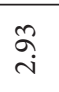 & 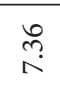 & 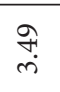 & $\stackrel{n}{i}$ & $\stackrel{\infty}{0}$ & 0 & $\begin{array}{l}\vec{\sigma} \\
\underset{\infty}{\infty}\end{array}$ & $\begin{array}{l}\mathbb{N} \\
\stackrel{0}{0}\end{array}$ & $\stackrel{5}{\circ}$ & $\begin{array}{l}\text { के } \\
\text { i }\end{array}$ & 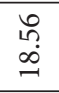 & $\begin{array}{l}\text { ô } \\
\text { i }\end{array}$ & $\stackrel{\vec{i}}{\vec{i}}$ & $\vec{m}$ & $\begin{array}{l}\infty \\
\infty \\
i \\
i\end{array}$ & $\underset{+}{\stackrel{J}{*}}$ \\
\hline & ๙্లু & $\infty_{\infty}^{\infty}$ & 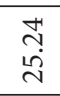 & $\stackrel{\partial}{2}$ & $\begin{array}{l}\hat{0} \\
\text { in }\end{array}$ & $\begin{array}{l}\hat{\tilde{O}} \\
\stackrel{0}{0}\end{array}$ & î & 0 & $\begin{array}{l}\infty \\
\infty \\
\end{array}$ & $\stackrel{m}{\tilde{0}}$ & $\stackrel{\infty}{\stackrel{\infty}{i}}$ & 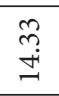 & $\begin{array}{l}\infty \\
\stackrel{\infty}{0} \\
0\end{array}$ & $\stackrel{\Re}{-}$ & $\vec{\sim}$ & $\underset{+}{\stackrel{H}{+}}$ & ָூ \\
\hline & స్ & 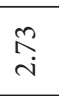 & $\stackrel{\text { I }}{\wedge}$ & $\stackrel{\infty}{i}$ & ָे & $\stackrel{\beta}{-}$ & 0 & $\begin{array}{l}\underset{\sim}{i} \\
\infty\end{array}$ & $\stackrel{\tilde{g}}{=}$ & $\stackrel{\infty}{0}$ & $\begin{array}{l}+ \\
0 \\
i\end{array}$ & $\underset{\jmath}{\stackrel{?}{2}}$ & \begin{tabular}{l}
\multirow{2}{*}{} \\
i
\end{tabular} & 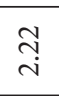 & $\stackrel{\infty}{i}$ & $\stackrel{5}{6}$ & $\stackrel{+}{\stackrel{\Delta}{0}}$ \\
\hline & 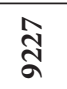 & $\stackrel{\infty}{\stackrel{\infty}{\rightarrow}}$ & $\stackrel{\text { oे }}{\text { Pे }}$ & 苫 & $\tilde{\text { İ }}$ & $\stackrel{0}{0}$ & 0 & $\begin{array}{l}\text { గ్ర } \\
\text { నু }\end{array}$ & 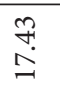 & ָิ & $\begin{array}{l}\infty \\
\infty \\
i\end{array}$ & $\begin{array}{l}\infty \\
m \\
i n \\
m\end{array}$ & $\begin{array}{l}\tilde{O} \\
\dot{i}\end{array}$ & $\stackrel{\hat{n}}{i}$ & $\underset{\text { तี }}{\text { in }}$ & 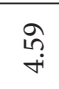 & $\stackrel{\text { I }}{\stackrel{I}{J}}$ \\
\hline & 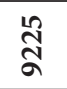 & $\hat{a}$ & $\stackrel{\infty}{\stackrel{\infty}{~}}$ & กิ & $\stackrel{\circ}{\circ}$ & 0 & 0 & $\begin{array}{l}\vec{b} \\
\stackrel{\alpha}{ }\end{array}$ & $\begin{array}{l}\underset{\sim}{N} \\
\stackrel{\sim}{*}\end{array}$ & $\stackrel{0}{0}$ & $\begin{array}{l}\infty \\
\stackrel{i}{i}\end{array}$ & $\begin{array}{l}\hat{N} \\
\stackrel{0}{0}\end{array}$ & $\begin{array}{l}\infty \\
\infty \\
i \\
i\end{array}$ & în & $\stackrel{H}{i}$ & iุ & $\stackrel{+}{\stackrel{N}{G}}$ \\
\hline \multirow{6}{*}{ 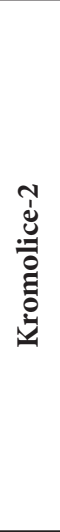 } & గू & $\begin{array}{l}\infty \\
\infty \\
0\end{array}$ & $\vec{i}$ & $\stackrel{\tilde{n}}{0}$ & $\stackrel{m}{0}$ & 0 & 0 & 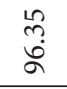 & $\begin{array}{l}\hat{0} \\
2 \\
-2\end{array}$ & $\stackrel{0}{0}$ & $\begin{array}{l}\stackrel{a}{ } \\
\text { i }\end{array}$ & 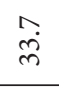 & $\begin{array}{l}\text { fo } \\
\text { i. } \\
\end{array}$ & $\underset{\text { In }}{\text { in }}$ & $\underset{m}{\stackrel{H}{*}}$ & 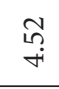 & $\begin{array}{l}\infty \\
\stackrel{0}{0} \\
\stackrel{+}{+}\end{array}$ \\
\hline & గิ & $\stackrel{\overbrace{}}{i}$ & $\begin{array}{l}\tilde{n} \\
\stackrel{n}{n}\end{array}$ & बे & $\vec{\sigma}$ & $\underset{-}{\hat{\sigma}}$ & 0 & 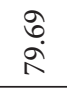 & $\overrightarrow{0}$ & ले & $\begin{array}{l}\hat{\infty} \\
i \\
i\end{array}$ & $\underset{m}{\stackrel{N}{N}}$ & $\begin{array}{l}0 \\
10 \\
i \\
1\end{array}$ & $\overrightarrow{\tilde{i}}$ & $\begin{array}{l}\infty \\
\stackrel{\infty}{i}\end{array}$ & $\underset{\stackrel{\infty}{\circ}}{\stackrel{\infty}{+}}$ & $\stackrel{\vec{\circ}}{\therefore}$ \\
\hline & $\frac{10}{2}$ & $\begin{array}{l}\infty \\
\stackrel{\infty}{i} \\
i\end{array}$ & 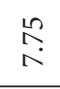 & $\stackrel{n}{i}$ & $\stackrel{\vec{n}}{-}$ & $\begin{array}{l}\infty \\
\infty \\
0 \\
0\end{array}$ & 0 & $\begin{array}{l}\stackrel{m}{+} \\
+ \\
\infty\end{array}$ & $\Xi$ & $\begin{array}{l}\mathscr{l} \\
\stackrel{1}{0}\end{array}$ & $\begin{array}{l}\hat{\infty} \\
i \\
i\end{array}$ & $\stackrel{\leftrightarrow}{\sim}$ & $\stackrel{\overrightarrow{0}}{i}$ & $\underset{i}{H}$ & $\vec{i}$ & $\stackrel{f}{+}$ & กี \\
\hline & $\frac{m}{2}$ & $\stackrel{\Re}{-}$ & $\stackrel{\infty}{\infty} \underset{+}{+}$ & $\underset{-}{F}$ & $\stackrel{\circ}{\circ}$ & $\begin{array}{l}\hat{0} \\
0\end{array}$ & 0 & $\begin{array}{l}\stackrel{H}{2} \\
\stackrel{2}{\alpha}\end{array}$ & 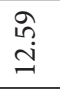 & तิ & $\begin{array}{l}\stackrel{1}{\infty} \\
\text { i }\end{array}$ & $\begin{array}{l}\vec{b} \\
\stackrel{i}{\mathbf{n}}\end{array}$ & $\begin{array}{l}H \\
10 \\
0 \\
1\end{array}$ & $\stackrel{\text { \& }}{i}$ & $\stackrel{ }{\stackrel{+}{-}}$ & $\begin{array}{l}\hat{\infty} \\
\dot{n}\end{array}$ & $\stackrel{n}{\stackrel{?}{\circ}}$ \\
\hline & $\frac{1}{2}$ & 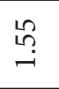 & $\stackrel{m}{\sim}$ & 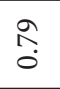 & $\stackrel{\sharp}{\circ}$ & 0 & 0 & $\begin{array}{l}\text { के } \\
\text { }\end{array}$ & $\begin{array}{l}\stackrel{2}{\hat{\alpha}} \\
10\end{array}$ & $\tilde{\widetilde{\sigma}}$ & $\begin{array}{l}\infty \\
\infty \\
i\end{array}$ & $\begin{array}{l}\hat{N} \\
\infty \\
\infty \\
\sim\end{array}$ & $\begin{array}{l}\infty \\
0 \\
0 \\
i\end{array}$ & $\stackrel{\text { ఫે }}{\text { i }}$ & $\stackrel{n}{i}$ & $\stackrel{\vartheta}{\vec{F}}$ & $\stackrel{\bullet}{\stackrel{\circ}{\wedge}}$ \\
\hline & $\begin{array}{l}\text { Oे } \\
\text { kू }\end{array}$ & $\stackrel{\circ}{\circ}$ & $\underset{i}{\stackrel{\Delta}{i}}$ & ઼ָ & กุ & 0 & 0 & 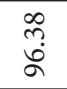 & $\stackrel{n}{0}$ & $\stackrel{1}{0}$ & $\begin{array}{l}\text { OD } \\
\text { i }\end{array}$ & 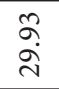 & $\stackrel{\widehat{N}}{i}$ & $\stackrel{n}{i}$ & $\underset{i}{F}$ & なू & 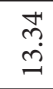 \\
\hline \multirow{8}{*}{ 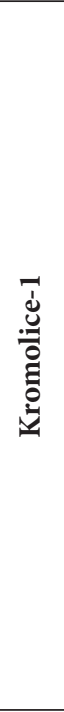 } & ڤे & $\overrightarrow{\tilde{n}}$ & $\vec{a}$ & 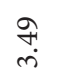 & $\begin{array}{l}\hat{\sigma} \\
i\end{array}$ & $\dot{o}$ & 0 & $\begin{array}{l}\text { f } \\
\stackrel{\infty}{\infty}\end{array}$ & त̄ & ֻٕ & $\begin{array}{l}\infty \\
\infty \\
i\end{array}$ & $\begin{array}{l}\text { ते } \\
\text { Oे }\end{array}$ & $\begin{array}{l}\vec{\sim} \\
\stackrel{i}{i}\end{array}$ & $\stackrel{\hat{m}}{i}$ & $\underset{\dot{j}}{\hat{i}}$ & ஸे & के \\
\hline & $\begin{array}{l}\infty \\
\infty \\
\infty \\
\infty\end{array}$ & $\stackrel{\vartheta}{\leftrightarrows}$ & $\underset{\sim}{\stackrel{N}{n}}$ & ஸి & $\stackrel{L}{0}$ & 0 & 0 & $\stackrel{m}{\stackrel{m}{+}}$ & $\overrightarrow{\vec{\lambda}}$ & $\overrightarrow{0}$ & $\stackrel{a}{i}$ & 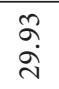 & $\begin{array}{l}\infty \\
\infty \\
i \\
i\end{array}$ & $\begin{array}{l}\stackrel{L}{h} \\
\text { in }\end{array}$ & $\begin{array}{l}\stackrel{L}{n} \\
i\end{array}$ & $\underset{\text { in }}{\text { in }}$ & $\stackrel{\infty}{\stackrel{\infty}{\lrcorner}}$ \\
\hline & ڤิ & $\begin{array}{l}B \\
i \\
i\end{array}$ & $\stackrel{m}{a}$ & $\stackrel{m}{m}$ & $\hat{i}$ & $\bar{a}$ & $\underset{r}{\mathbb{n}}$ & $\begin{array}{l}\stackrel{\infty}{\infty} \\
\stackrel{1}{\wedge}\end{array}$ & $\begin{array}{l}\tilde{N} \\
\stackrel{0}{0}\end{array}$ & m̊ & $\begin{array}{l}\vec{\infty} \\
i\end{array}$ & 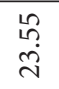 & $\stackrel{\overbrace{}}{\tilde{0}}$ & $\stackrel{\hat{m}}{i}$ & 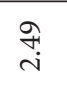 & $\stackrel{\widehat{\Im}}{+}$ & 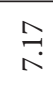 \\
\hline & ڤે & $\underset{i}{\stackrel{\sim}{i}}$ & $\begin{array}{l}\hat{L} \\
\stackrel{n}{1}\end{array}$ & $\underset{i}{\stackrel{\Delta}{i}}$ & $\stackrel{\tilde{\beta}}{-}$ & $\overrightarrow{0}$ & 0 & $\begin{array}{l}0 \\
+1 \\
\infty \\
\infty\end{array}$ & $\begin{array}{l}\hat{n} \\
\stackrel{n}{\mathrm{~N}}\end{array}$ & $\stackrel{\text { İ }}{0}$ & $\begin{array}{l}\infty \\
\infty \\
i\end{array}$ & $\underset{\stackrel{\sim}{\vec{\lambda}}}{ }$ & $\underset{+}{\stackrel{+}{+}}$ & $\stackrel{\mathscr{n}}{\sim}$ & $\begin{array}{l}\stackrel{H}{0} \\
i\end{array}$ & $\begin{array}{l}\stackrel{\infty}{\infty} \\
+ \\
+\end{array}$ & $\begin{array}{c}\stackrel{0}{L} \\
\infty \\
\infty\end{array}$ \\
\hline & 今े & $\underset{i}{\stackrel{\circ}{ }}$ & $\underset{+}{\stackrel{H}{+}}$ & $\stackrel{\infty}{\infty}$ & ঙิ & กี & 0 & $\begin{array}{l}\vec{\infty} \\
\infty \\
\infty\end{array}$ & 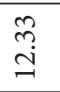 & $\stackrel{0}{0}$ & $\begin{array}{l}\underset{\infty}{\infty} \\
i\end{array}$ & $\begin{array}{l}\stackrel{+}{\infty} \\
\sim \\
\dot{\lambda}\end{array}$ & $\begin{array}{l}\text { m} \\
\stackrel{1}{1}\end{array}$ & $\overrightarrow{\text { ते }}$ & $\begin{array}{l}\stackrel{0}{i} \\
\text { i }\end{array}$ & $\overrightarrow{\vec{n}}$ & $\underset{\sim}{\stackrel{H}{n}}$ \\
\hline & 茫 & $\stackrel{\leftrightarrow}{-}$ & $\stackrel{\llcorner}{+}$ & 藏 & $\stackrel{7}{\circ}$ & 0 & 0 & 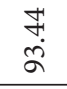 & $\begin{array}{l}\text { oे. } \\
\text { i }\end{array}$ & $\stackrel{\infty}{\stackrel{\infty}{0}}$ & $\hat{i}$ & $\begin{array}{l}\hat{b} \\
\hat{m}\end{array}$ & $\begin{array}{l}\bar{n} \\
0 \\
i\end{array}$ & $\begin{array}{l}\vec{n} \\
\stackrel{i}{i}\end{array}$ & 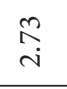 & $\stackrel{\circ}{\stackrel{2}{n}}$ & $\sigma$ \\
\hline & के & ڤે & $\stackrel{0}{\underset{+}{+}}$ & in & $\stackrel{1}{0}$ & 0 & 0 & nn & $\begin{array}{l}\vec{\infty} \\
\dot{0} \\
-\end{array}$ & $\stackrel{10}{0}$ & $\begin{array}{l}\stackrel{\infty}{0} \\
\text { i }\end{array}$ & $\stackrel{\sim}{\tilde{\sim}}$ & 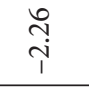 & $\stackrel{\widetilde{\sim}}{\mathrm{i}}$ & $\begin{array}{l}\text {. } \\
\text { i }\end{array}$ & $\sqrt{6}$ & भ̊ \\
\hline & $\begin{array}{l}\infty \\
\infty \\
\infty\end{array}$ & $\stackrel{\sim}{\sim}$ & $\stackrel{H}{i}$ & $\overrightarrow{\vec{i}}$ & 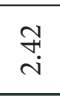 & fै & 0 & \begin{tabular}{c} 
ले \\
\multirow{\sigma}{*}{}
\end{tabular} & $\stackrel{\infty}{=}$ & त̃ & $\begin{array}{l}\vec{\infty} \\
i\end{array}$ & $\begin{array}{l}\stackrel{n}{\hat{n}} \\
\stackrel{\sim}{N}\end{array}$ & $\begin{array}{l}0 \\
\infty \\
i \\
i\end{array}$ & f્ & $\stackrel{\infty}{\stackrel{\infty}{i}}$ & $\stackrel{\Re}{\hat{\sigma}}$ & 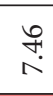 \\
\hline & & \multicolumn{13}{|c|}{ LO-0.J!W } & \multicolumn{3}{|c|}{ [\%] YWN } \\
\hline & & \multicolumn{8}{|c|}{$\delta^{\circ}$} & \multirow[b]{2}{*}{ 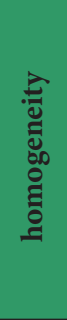 } & \multirow{2}{*}{ 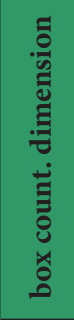 } & \multirow[b]{2}{*}{ 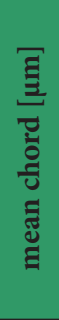 } & \multirow[b]{2}{*}{ 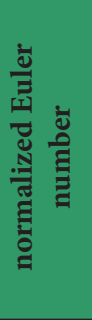 } & \multirow[b]{2}{*}{ 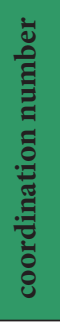 } & \multirow[b]{2}{*}{$\overline{\underline{a}}$} & \multirow[b]{2}{*}{$\tilde{\underline{z}}$} & \multirow[b]{2}{*}{$\hat{\underline{\theta}}$} \\
\hline & 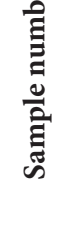 & - & $\exists$ & $\Xi$ & $\geq$ & $>$ & 5 & $\overline{7}$ & 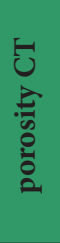 & & & & & & & & \\
\hline & & - & $N$ & $m$ & $r$ & in & 0 & $n$ & $\infty$ & $a$ & 요 & $\exists$ & $\simeq$ & 2 & $\Xi$ & $\stackrel{10}{2}$ & $\stackrel{\bullet}{\sigma}$ \\
\hline
\end{tabular}




\begin{tabular}{|c|c|c|c|c|c|c|c|c|c|c|c|c|c|c|}
\hline \multirow{7}{*}{ 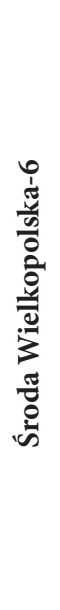 } & 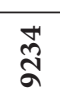 & $\underset{\infty}{\stackrel{+}{\infty}}$ & 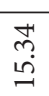 & $\begin{array}{l}\vec{\sigma} \\
\dot{0}\end{array}$ & $\underset{\mathscr{U}}{\tilde{\sigma}}$ & 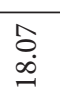 & $\begin{array}{l}\tilde{N} \\
\stackrel{2}{2}\end{array}$ & $\stackrel{\widetilde{\Xi}}{=}$ & $\begin{array}{l}0 \\
0 \\
0\end{array}$ & $\stackrel{\substack{0 \\
0}}{0}$ & $\stackrel{\widehat{0}}{0}$ & 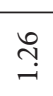 & $\stackrel{\vec{T}}{\rightarrow}$ & $\underset{\sim}{\stackrel{H}{i}}$ \\
\hline & $\stackrel{\widetilde{\Omega}}{\Omega}$ & 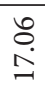 & $\stackrel{\Re}{\stackrel{\Re}{+}}$ & 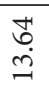 & 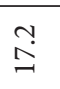 & $\begin{array}{l}\hat{b} \\
\infty\end{array}$ & $\begin{array}{l}\text { त్ } \\
\stackrel{\text { ¿े }}{ }\end{array}$ & $\stackrel{\circ}{\circ}$ & $\stackrel{n}{20}$ & $\begin{array}{l}\text { ț } \\
0\end{array}$ & $\stackrel{7}{0}$ & $\stackrel{乛}{-}$ & I & 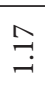 \\
\hline & ๙ู & $\vec{m}$ & 으 & 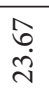 & $=$ & ה્م & $\begin{array}{l}\stackrel{0}{2} \\
\text { 子̇ }\end{array}$ & $\widetilde{-}$ & तु & ?? & 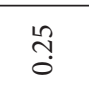 & $\stackrel{\text { fq }}{\rightarrow}$ & $\vec{m}$ & 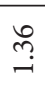 \\
\hline & ్ָల్ & $\hat{\alpha}$ & 点 & 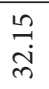 & $\stackrel{n}{n}$ & 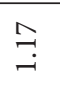 & 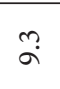 & 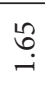 & $\stackrel{0}{0}$ & $\stackrel{\infty}{m}$ & : & 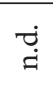 & $\stackrel{ت}{\dot{g}}$ & $\stackrel{ت}{\dot{g}}$ \\
\hline & $\underset{\text { స్ }}{ }$ & $\stackrel{2}{=}$ & $\vec{I}$ & 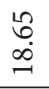 & $\hat{\ddot{g}}$ & ڤे. & $\begin{array}{l}\text { Pे } \\
\text { in }\end{array}$ & $\stackrel{m}{i}$ & $\stackrel{\leftrightarrow}{\stackrel{H}{0}}$ & ?ै & 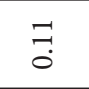 & $\stackrel{f}{\rightarrow}$ & $\stackrel{\overbrace{}}{\overbrace{}}$ & $\vec{m}$ \\
\hline & สิ & $\stackrel{\text { L }}{\circ}$ & 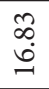 & $\begin{array}{l}\stackrel{\text { L }}{\Xi} \\
\end{array}$ & $\stackrel{\sim}{\infty}$ & 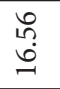 & $\vec{\Xi}$ & $\stackrel{\infty}{\leftrightarrows}$ & $\exists$ & $\begin{array}{l}\vec{\infty} \\
0 \\
0\end{array}$ & $\stackrel{\circ}{0}$ & $\fallingdotseq$ & $\stackrel{?}{\leftrightarrows}$ & 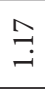 \\
\hline & สู & $\begin{array}{l}\vec{\Delta} \\
\text { बे }\end{array}$ & 㫄 & $\begin{array}{l}\stackrel{\tilde{O}}{\mathrm{j}} \\
\end{array}$ & ֻั & $\begin{array}{l}0 \\
\hat{m} \\
\end{array}$ & $\stackrel{\infty}{\stackrel{\sim}{*}}$ & 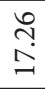 & $\overrightarrow{\mathrm{i}}$ & $\begin{array}{c}\infty \\
\infty \\
\infty \\
0\end{array}$ & $\stackrel{n}{\circ}$ & $\underset{ت}{ت}$ & $\stackrel{\mathscr{m}}{\rightarrow}$ & $\stackrel{m}{=}$ \\
\hline \multirow{6}{*}{ 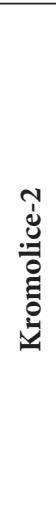 } & $\begin{array}{l}\mathbb{Z} \\
\text { ถू }\end{array}$ & 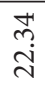 & $\stackrel{2}{\Omega}$ & $\begin{array}{l}\stackrel{8}{0} \\
+\end{array}$ & $\begin{array}{l}m \\
\stackrel{m}{n}\end{array}$ & $\begin{array}{l}\overrightarrow{7} \\
\dot{\theta}\end{array}$ & $\begin{array}{l}\stackrel{શ}{i} \\
\stackrel{i}{n}\end{array}$ & 兽 & तે & $\stackrel{\Re}{o}$ & ? & $\stackrel{0}{=}$ & $\underset{ت}{\leftrightarrows}$ & $\stackrel{0}{\rightarrow}$ \\
\hline & గ్ & $\begin{array}{l}\bar{n} \\
\stackrel{\text { nf }}{+}\end{array}$ & 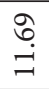 & $\begin{array}{l}\overrightarrow{7} \\
2 \\
2\end{array}$ & $\hat{\vec{g}}$ & $\hat{m}$ & $\begin{array}{l}\infty \\
\infty \\
\infty \\
\end{array}$ & $\stackrel{\circ}{\circ}$ & m. & $\hat{o}$ & $\stackrel{H}{0}$ & ت্] & $\stackrel{\mathbb{S}}{\rightarrow}$ & $\stackrel{\leftrightarrow}{\rightarrow}$ \\
\hline & $\frac{n}{k n}$ & 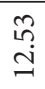 & $\stackrel{\beta}{\circ}$ & 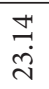 & น? & $\underset{i}{\mathbb{N}}$ & $\begin{array}{l}\infty \\
\stackrel{\infty}{2} \\
\end{array}$ & fि & F! & ㅊ. & $\tilde{\overbrace{}}$ & $\stackrel{m}{m}$ & $\stackrel{\widehat{m}}{\rightarrow}$ & $\stackrel{\overbrace{}}{\rightrightarrows}$ \\
\hline & $\frac{m}{2}$ & $\begin{array}{l}\hat{\theta} \\
\text { فे }\end{array}$ & 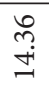 & $\begin{array}{l}\text { ț } \\
\stackrel{0}{\circ}\end{array}$ & $\stackrel{\stackrel{H}{J}}{J}$ & $\stackrel{n}{m}$ & 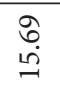 & $\approx$ & $\vec{\infty}$ & $\stackrel{\infty}{\circ}$ & $\tilde{\text { ஸे }}$ & ঙ્গ & 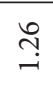 & $\stackrel{\sim}{\rightarrow}$ \\
\hline & $\frac{1}{2}$ & $\stackrel{\vec{m}}{\stackrel{H}{*}}$ & 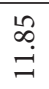 & 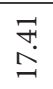 & $\stackrel{\rightleftarrows}{+}$ & ô & $\begin{array}{l}\infty \\
\stackrel{\infty}{0} \\
\dot{\sim}\end{array}$ & $\stackrel{n}{n}$ & $\begin{array}{l}\infty \\
\stackrel{\infty}{0} \\
0\end{array}$ & 종 & $\stackrel{\text { Oे }}{0}$ & $\stackrel{\overbrace{}}{\stackrel{n}{-}}$ & $\stackrel{\overbrace{}}{\rightrightarrows}$ & $\stackrel{\Upsilon}{-1}$ \\
\hline & $\begin{array}{l}\hat{\partial} \\
\text { Hू }\end{array}$ & $\hat{\stackrel{\sim}{े}}$ & 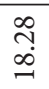 & 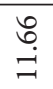 & $\stackrel{m}{\sim}$ & $\begin{array}{l}\hat{0} \\
\hat{D} \\
\end{array}$ & $\begin{array}{l}\stackrel{2}{\sim} \\
\vec{\lambda}\end{array}$ & $\begin{array}{l}\text { ָ̃ } \\
\underset{\exists}{*}\end{array}$ & $\stackrel{0}{0}$ & $\stackrel{\widehat{A}}{\hat{0}}$ & $\ddot{\circ}$ & $\stackrel{\overbrace{}}{\rightrightarrows}$ & $\stackrel{\rightrightarrows}{\rightrightarrows}$ & 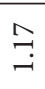 \\
\hline \multirow{8}{*}{ 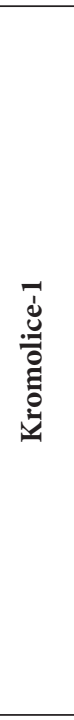 } & ठి & 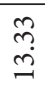 & 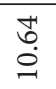 & $\begin{array}{l}\hat{i} \\
\stackrel{\text { in }}{1}\end{array}$ & $\stackrel{\vec{m}}{\rightarrow}$ & 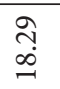 & $\begin{array}{l}\tilde{D} \\
\stackrel{J}{J}\end{array}$ & $\stackrel{\overbrace{}}{\sim}$ & \begin{tabular}{l}
$\stackrel{0}{\infty}$ \\
\hdashline \\
0
\end{tabular} & 尽 & $\stackrel{\text { 능 }}{0}$ & $\stackrel{\text { }}{\rightarrow}$ & $\stackrel{f}{\text { f }}$ & $\stackrel{\text { Î }}{\rightarrow}$ \\
\hline & $\begin{array}{l}\infty \\
\stackrel{\circ}{\circ}\end{array}$ & 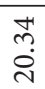 & 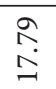 & 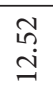 & $\stackrel{\bumpeq}{\bumpeq}$ & ஸ̂. & $\begin{array}{l}\text { ñ. } \\
\text { }\end{array}$ & $\begin{array}{l}\stackrel{巳}{+} \\
\stackrel{+}{ \pm}\end{array}$ & Ĥt & $\begin{array}{l}\vec{\sigma} \\
0 \\
0\end{array}$ & $\stackrel{10}{0}$ & $\underset{ت}{\leftrightarrows}$ & $\stackrel{\text { If }}{\rightarrow}$ & $\stackrel{0}{=}$ \\
\hline & ถู & 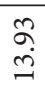 & $\underset{ت}{\stackrel{H}{二}}$ & $\stackrel{\Omega}{\beth}$ & 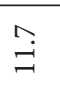 & $\begin{array}{l}\text { in } \\
\text { in }\end{array}$ & 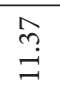 & $\stackrel{\widetilde{n}}{i}$ & $\stackrel{10}{0}$ & $\ddot{0}$ & $\stackrel{\text { ô }}{\circ}$ & 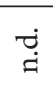 & $\stackrel{?}{\rightarrow}$ & $\stackrel{\mathbb{S}}{\stackrel{H}{H}}$ \\
\hline & बे & $\begin{array}{l}\text { బू } \\
\text { فn }\end{array}$ & $\underset{d}{\stackrel{F}{~}}$ & $\begin{array}{l}\widehat{\sigma} \\
\stackrel{2}{2}\end{array}$ & $\hat{a}$ & $\begin{array}{l}\stackrel{R}{0} \\
i\end{array}$ & $\underset{\sim}{\stackrel{\sim}{*}}$ & $\stackrel{\overbrace{}}{\stackrel{i}{i}}$ & $\stackrel{10}{0}$ & $\stackrel{m}{H}$ & ஸै? & تُ & $\stackrel{\mathbb{S}}{\mathrm{I}}$ & $\stackrel{\overbrace{}}{\rightarrow}$ \\
\hline & 离 & î & 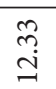 & $\begin{array}{l}\vec{m} \\
\stackrel{\infty}{\infty}\end{array}$ & $\stackrel{\vec{\sigma}}{\circ}$ & $\stackrel{\vec{\lambda}}{\vec{i}}$ & 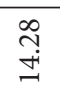 & $\underset{m}{m}$ & $\begin{array}{l}\stackrel{0}{0} \\
\stackrel{2}{0}\end{array}$ & 苞 & $\stackrel{10}{0}$ & $\stackrel{+}{\stackrel{m}{-}}$ & 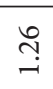 & ণ্ডী \\
\hline & F্ & $\stackrel{\vec{\sigma}}{\vec{a}}$ & 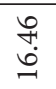 & $\overrightarrow{\stackrel{J}{J}}$ & $\approx$ & $\stackrel{n}{=}$ & $\stackrel{m}{2}$ & $\stackrel{\text { f }}{\mathrm{I}}$ & $\stackrel{\vec{\sigma}}{-}$ & શิ & đ̋ & $\stackrel{n}{\longrightarrow}$ & $\stackrel{\mathscr{2}}{\rightleftarrows}$ & $\stackrel{\overbrace{}}{\stackrel{\longrightarrow}{ت}}$ \\
\hline & ळे & $\vec{\circ}$ & $\begin{array}{l}0 \\
\stackrel{0}{0}\end{array}$ & $\begin{array}{l}\hat{\Phi} \\
\text { aे }\end{array}$ & $\stackrel{\circ}{\stackrel{\sim}{\sim}}$ & $\begin{array}{l}\infty \\
\infty \\
\hat{n}\end{array}$ & $\begin{array}{l}\Omega \\
\Omega\end{array}$ & 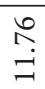 & $\stackrel{\overbrace{}}{-}$ & 令 & $\stackrel{n}{0}$ & $\stackrel{1}{7}$ & $\stackrel{\Upsilon}{7}$ & $\stackrel{\overbrace{}}{ت}$ \\
\hline & $\begin{array}{l}\infty \\
\tilde{\infty}_{\infty}\end{array}$ & 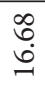 & $\stackrel{\text { I }}{\sharp}$ & 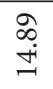 & $\underset{ٌ}{\tilde{O}}$ & 年 & ֶָ. & $\underset{\infty}{\infty}$ & $\stackrel{m}{3}$ & $\begin{array}{l}\infty \\
: \\
0\end{array}$ & $\overrightarrow{0}$ & $\stackrel{\overbrace{}}{\stackrel{\overbrace{}}{\sim}}$ & $\stackrel{\widehat{ }}{-}$ & $\stackrel{\leftrightarrow}{\rightarrow}$ \\
\hline & \multirow{4}{*}{ 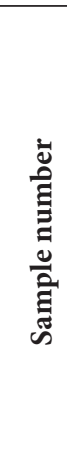 } & \multicolumn{3}{|c|}{ [\%] yWN } & $\dot{a}^{\circ}$ & $\overline{\mathrm{A}}$ & \multicolumn{4}{|c|}{$\stackrel{5}{2}$} & 1 & \multirow{2}{*}{\multicolumn{3}{|c|}{ 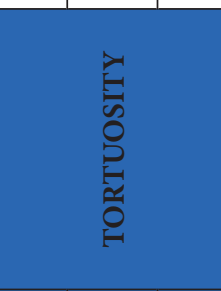 }} \\
\hline & & 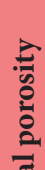 & 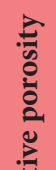 & 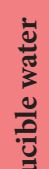 & 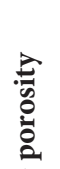 & 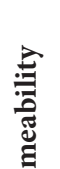 & 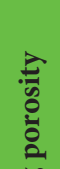 & $\begin{array}{l}\underline{\Xi} \\
0 \\
\hat{b}\end{array}$ & 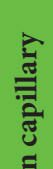 & $\begin{array}{l}\underline{E} \\
\hat{\hat{\theta}} \\
\hat{\otimes}\end{array}$ & 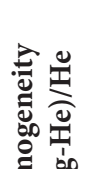 & & & \\
\hline & & 9 & छัँ &. & 7 & $\approx$ & $I$ & 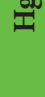 & $\Xi$ & 气 & & $\star$ & $\lambda$ & $\mathbf{N}$ \\
\hline & & $\cong$ & $\stackrel{\infty}{\sim}$ & 2 & 오 & $\vec{\lambda}$ & $\tilde{\text { ก }}$ & $\tilde{\lambda}$ & $\Xi$ & $\stackrel{\text { L }}{\mathrm{N}}$ & i & $\hat{\lambda}$ & 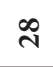 & ते \\
\hline
\end{tabular}




\section{Table 8}

Summary correlation matrix

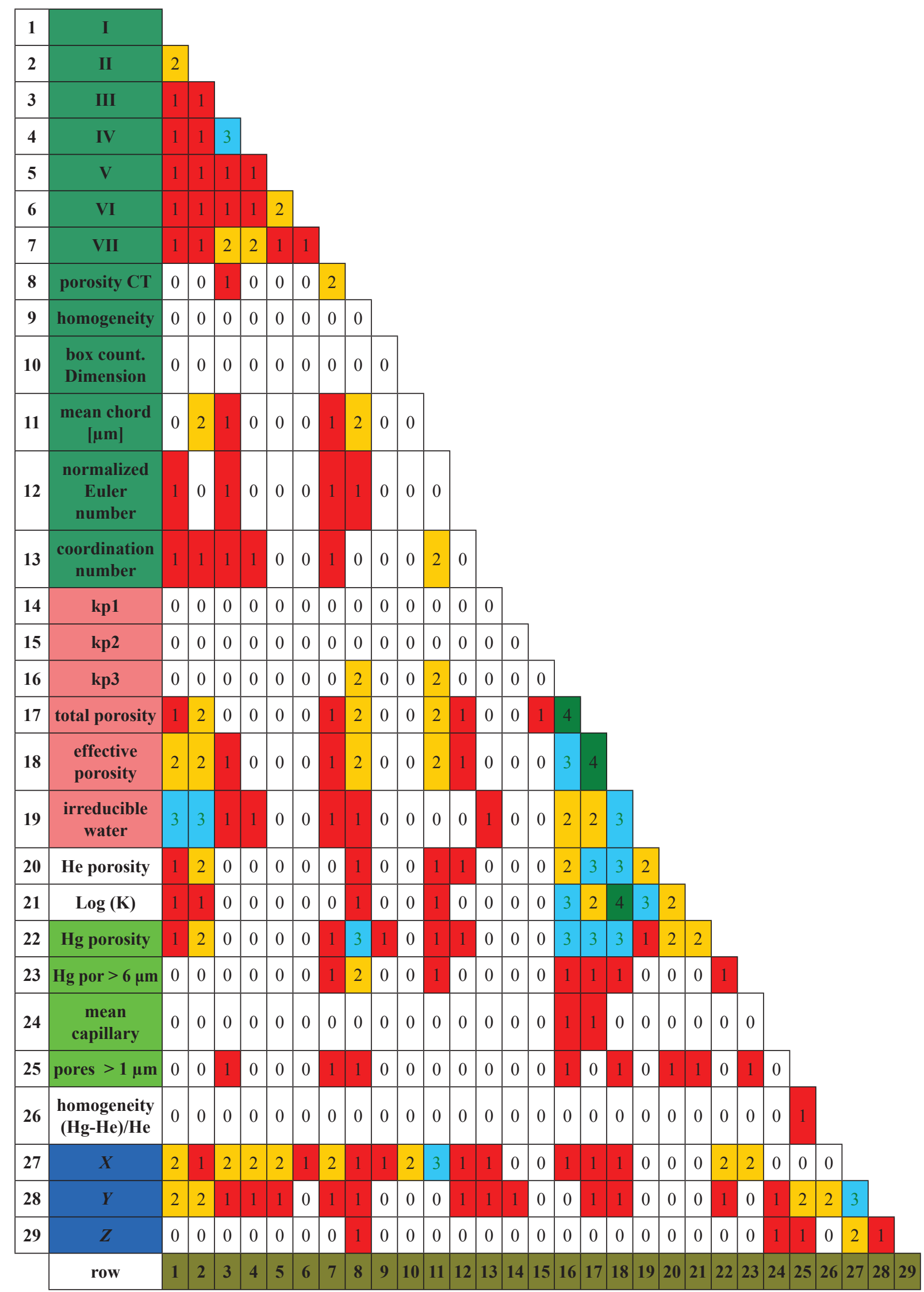




\section{Mercury injection capillary pressure (MICP)}

The mercury injection capillary pressure (MICP) measured the pressure that was necessary to inject non-wetting liquid (mercury) into the pore space of a sample (Tsakiroglu \& Payatakes 2000). The relationship between pressure and volume of mercury penetrating porous space is the basis for calculating several parameters. In this paper, the following parameters obtained from MICP were used: effective porosity [\%], effective porosity for pore throats greater than $6 \mu \mathrm{m}$ [\%], diameter of an average capillary and contribution of pores with diameter greater than $1 \mu \mathrm{m}[\%]$.

\section{Nuclear magnetic resonance (NMR)}

The nuclear magnetic resonance (NMR) is a technique of porosity determination, which was introduced to laboratory rock investigations, later than other porosity measurement methods (pycnometry or porosimetry) (Akkurt et al. 1995, Xiao et al. 2008, Klaja \& Gąsior 2010). This technique used particular features of magnetic moment and spin that were characteristic of nuclei of some elements including hydrogen (Straley et al. 1997, Coates et al. 1999). With the use of widely known laboratory measurement technique, CPMG, the transverse relaxation time, $T_{2}$, of hydrogen nuclei in rocks was determined. The distribution and amplitude of $T_{2}$ depended on pore size in rock samples, and on how the pores were saturated with free media (water) and media bonded by capillary force and contained in shales. The samples were completely saturated with brine, whose mineralization corresponded to that of reservoir waters from the Fore-Sudetic Monocline. In further study the following parameters obtained with NMR were used: $k_{p 1}$ - irreducible water [\%], $k_{p 2}$ - capillary water [\%], $k_{p 3}$ - free water [\%], total porosity [\%], effective porosity [\%] and irreducible water saturation [\%].

\section{Helium pycnometer porosity}

Helium pycnometer belongs to the class of gas pycnometers, standard laboratory devices used for measuring the density and determining total porosity (www.micromeritics.com).

\section{Permeameter - physical permeability}

Permeability is a property responsible for fluid/ gas flow in porous rock formation. The parameter can be directly obtained only in a laboratory, using permeameters. There is a great variety of measuring facilities. In the paper physical permeability, determined by a TEMCO permeameter, was used in the grouping of reservoir properties.

\section{RESULTS AND THEIR INTERPRETATION}

Results of the investigations presented in tables 5-8 showed that the examined parameters of samples from Region 2 were definitely different than the parameters measured for samples from other regions. Average value of the NMR effective porosity for Region 2 was $13.85 \%$, while for Regions 1 and 3 , the values were equal to $6.45 \%$ and $8.32 \%$, respectively. A similar trend was observed for total porosity, obtained by helium pycnometry and mercury porosimetry. In addition, most parameters obtained from the micro-CT method were very different for Region 2 than for other areas. The average contribution of subsystems classified to class VII of pore volume was $85.24 \%$ for that region. It amounted to 30\% in Region 3, and 19\% in Region 1. A threefold higher average value of porosity was also obtained from the micro-CT measurements.

The mean chord length of $26.11 \mu \mathrm{m}$ was largest for samples of Region 2. It was equal to about $17.5 \mu \mathrm{m}$ for other regions. Analysis of tortuosity of pore channels also showed that samples from Region 2 had the best communicated pore structure and the highest average value of tortuosity. A tortuosity value of zero was assigned to samples in which there were no communications between pores in an analyzed direction. Therefore, higher average values of tortuosity were not considered to represent structures with more tortuous pore structure. Instead, they indicated a considerable number of samples with pore communications in the analyzed direction.

Similar behavior was observed for the box counting dimension. The maximum value of 2.85 was obtained for Region 2. It was equal to 2.82 for Region 3 and 2.63 for Region 1. Values of normalized Euler number also differentiated the examined regions. The analyzed parameters were organized in the following increasing sequence: Region 1, 3, and 2. It was also concluded that 
petrophysical properties evaluated by computed microtomography displayed the same trends as those measured by standard methods. This statement was the basis for selecting rock samples to more advanced individual examination, with the use of microtomography.

In the next stage of data analysis, mutual relationships (correlations) between the obtained parameters were found. Four correlation matrices were created, and calculations were made separately for sets containing all samples and individual regions. Furthermore, cells with determination coefficients greater than 0.6 were selected, and the summary correlation matrix was created to show which parameters were most often related (Tab. 8).

The values in the matrix cells were calculated as follows:

0 - when in no matrix, the absolute value of determination coefficient was found to be greater than 0.6 in the given cell,

1 - when in one matrix, the absolute value of determination coefficient was found to be greater than 0.6 in the given cell,

2 - when in two matrices, the absolute value of determination coefficient was found to be greater than 0.6 in the given cell,

3 - when in three matrices, the absolute value of determination coefficient was found to be greater than 0.6 in the given cell,

4 - when in four matrices, the absolute value of determination coefficient was found to be greater than 0.6 in the given cell.

The summary correlation matrix (Tab. 8) shows that each set type (all samples or individual regions separately) always revealed the correlation between the following parameters obtained by different methods: class I volume vs. irreducible water volume; porosity obtained from the micro-CT method vs. porosity from the mercury injection capillary pressure method; free water volume and effective porosity vs. porosity obtained from the mercury injection capillary pressure method and free water volume vs. permeability. In three cases, the correlation was obtained between: porosity from micro-CT vs. free water volume, vs. total porosity, vs. effective porosity from NMR, mean chord length vs. free water volume, and total porosity vs. effective porosity from NMR, porosity from helium pycnometer vs. free water volume, and total porosity vs. effective porosity from NMR, NMR-based effective porosity vs. pycnometer-based total porosity, vs. porosity from mercury injection capillary pressure, and effective porosity vs. permeability. Single cases of correlation or no correlation were observed between other parameters. Explanation of high correlation or poor relationship between parameters listed above is based on physical phenomena being the basis of fluid flow in porous space of clastic rocks and the properties of rocks. Previous chapters presented different properties of rocks in the study and selected characteristics of the used methods to underline specific features on both sides. For instance, class I volume and irreducible water volume revealed a high correlation, because both parameters are related to the group of smallest pores micro-CT porosity is related to all pores, and MICP porosity is a sum of connected pores intruded by mercury. High correlation between these two parameters means that the level of difference between two porosities is constant in the studied population (Tabs $5-8$ ). The relationship between free water volume and effective porosity (NMR origin) and MICP porosity is obvious on the basis of the physical basis of both methods. Connection between mean chord length and volume of pores in the defined subsystems was presented in Table 3. It was confirmed by its correlation with free water volume. In the correlation matrix (Tab. 8), the precise values of the determination coefficients were not important. It was important that the correlation was high. Table 8 enabled recognition of high correlation relationships between parameters considered in the total data set and in data sets related to regions. High determination coefficients for the full data set (containing all samples) were obtained for relationships between porosity calculated from mercury porosimetry and the micro-CT method $\left(R^{2}=0.86\right)($ Fig. 14A) and between NMR effective porosity and mean chord length from the micro-CT method $\left(R^{2}=0.72\right)$ (Fig. 14B).

High correlations $\left(R^{2}=0.79\right)$ were observed between NMR effective porosity and porosity from the micro-CT method (Fig. 15A); for NMR effective porosity and normalized Euler number (Fig. 15B), for samples from Region 2. 


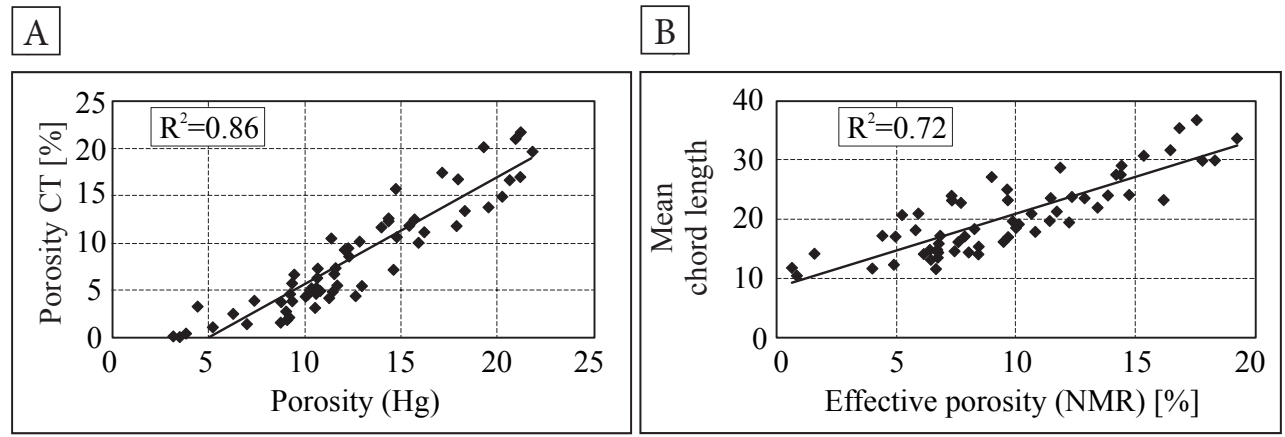

Fig. 14. Relationships between selected parameters for the full data set

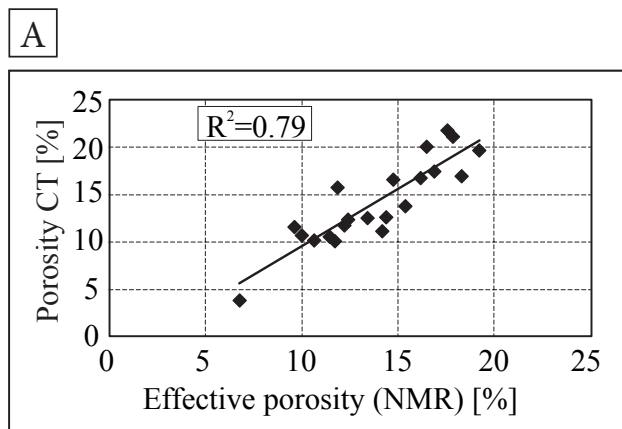

$\mathrm{B}$

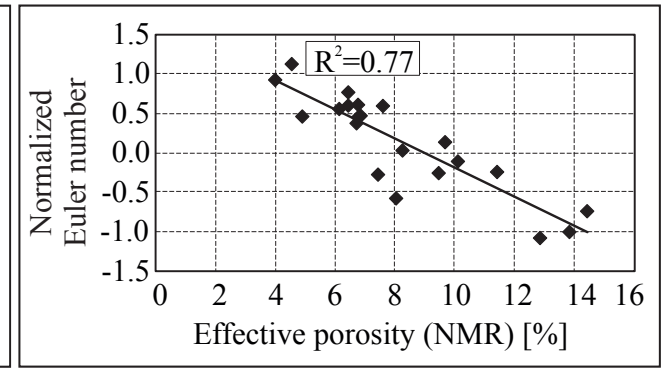

Fig. 15. Relationships between selected parameters for Region 2

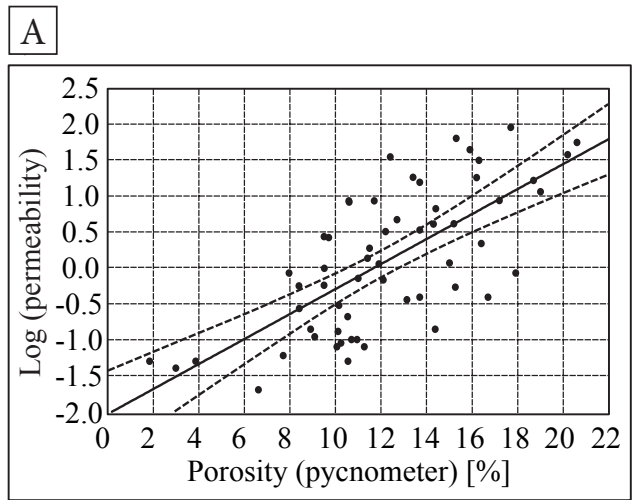

B

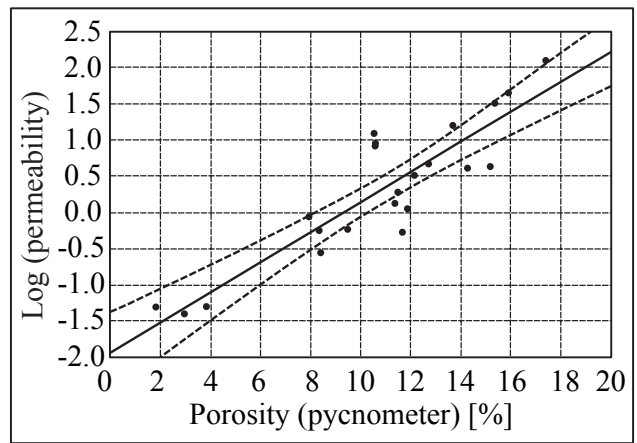

Fig. 16. Dispersion plots of permeability vs. helium pycnometer porosity: A) full data set; B) data set from Region 1

The calculated correlations formed the basis for deducting on some parameters based on other ones. For example, the normalized Euler number is a parameter describing communications between free spaces of irregular shape in rock (pores with different tortuosity), and can be determined on the basis of effective porosity based on NMR measurements. First of all, such an approach enhanced the qualitative information of the pore space. In addition, it led to the parametrization of quantities that are difficult to be analytically calculated or experimentally measured, e.g. tortuosity of pore channels. Correlations with high coefficients were the basis for predicting certain parameters.
Prediction of absolute permeability based on porosity measured with the use of helium pycnometer is presented in Figure 16A. The determination coefficient for full data set is 0.53 and the estimation error is 0.71 . The same parameters which correlate well for samples from Region 1 are presented in Figure 16B, with a higher correlation coefficient $(0.85)$ and smaller estimation error (0.44).

Results of the multivariate regression analysis of permeability, obtained from the micro-CT method (Tab. 9), proved that the mean chord length and normalized Euler number contributed significant information to the model. Permeability $(K)$ can be predicted using formula: 


$$
\begin{aligned}
K= & b_{0}+b_{1} \cdot \text { porosity_CT }+b_{2} \cdot \text { mean_chord }+ \\
+ & b_{3} \cdot \text { normalized_Euler_number }+ \\
& +b_{4} \cdot \text { coordination_number }
\end{aligned}
$$

where:

$K$ - permeability (dependent variable), $b_{0}-b_{4}-$ coefficients in the regression equation,

porosity CT - coordination number (independent variables).
The high correlation between the predicted values of permeability (on the basis of micro-CT porosity model) vs. the observed values of permeability confirmed that the model can be used for prediction (Fig. 17).

It is also interesting to point to the connection between computed X-ray microtomography results related to pore subsystem classification, the ability of fluid flow in the pore space and the tortuosity of porous network vs. XRD outcomes relating to mineral composition.

\begin{tabular}{|c|c|c|c|c|}
\hline \multirow{2}{*}{$N=60$} & \multicolumn{4}{|c|}{$\begin{array}{c}R^{2}=0.63, \text { adjusted } R^{2}=0.60 \\
\text { standard error of estimate: } 0.65\end{array}$} \\
\hline & $b$ & standard error with $b$ & $t$ & $p$ \\
\hline Constant & $b_{0}=-1.06786$ & 0.764406 & -1.39698 & 0.168030 \\
\hline Porosity CT & $b_{1}=-0.05601$ & 0.033746 & -1.65984 & 0.102639 \\
\hline Mean chord & $b_{2}=0.14003$ & 0.028628 & 4.89151 & 0.000009 \\
\hline Normalized Euler number & $b_{3}=-0.51643$ & 0.164345 & -3.14233 & 0.002699 \\
\hline Coordination number & $b_{4}=-0.52906$ & 0.428716 & -1.23405 & 0.222430 \\
\hline
\end{tabular}

Table 9

Results of the multivariate regression

Explanations: $R^{2}$ - coefficient of multiple determination which can measure the reduction in the total variation of the dependent variable, as a result of multiple independent variables; $R^{2}$ adjusted - the same as previous, but takes into consideration the degree of freedom; the standard error of estimate measures the dispersion of observed values around the regression line; $b$-coefficient representing individual contribution of the independent variable to the prediction of the dependent variable; $t$-value and resulting $p$-value are used to test the relationship between dependent and independent variables.

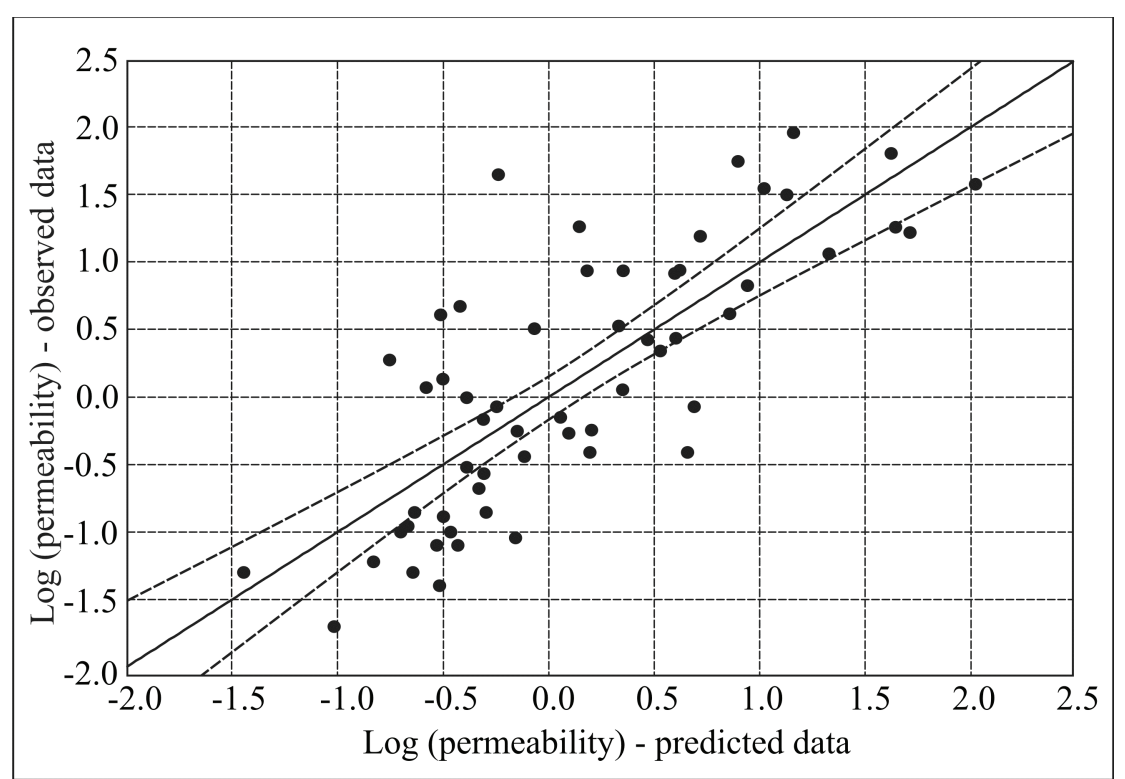

Fig. 17. Dispersion plot of predicted values of permeability vs. observed values of permeability; confidence intervals are on the level of 0.95 
Samples from Region 2, revealing the best reservoir properties, characterize the highest average volume of quartz and the lowest volume of clay minerals and micas. In the literature, it is mentioned that one can combine the micro CT and XRF techniques. The example describing the results of the sandstone investigations is very promising (Mutina \& Bruyndonckx 2013). Furthermore, it is possible to expect that using the rocks from the same location makes it possible to track the relationships between morphological properties and chemical compounds in a more robust and straightforward manner. The technology of investigations ( $5 \mathrm{~mm}$ diameter sandstone samples) is similar to that presented in this paper. As such, future investigations using computed X-ray microtomography can be planned.

\section{CONCLUSIONS}

Results obtained by using the computed X-ray microtomography and NMR experiment confirmed that the examined petrophysical parameters of the samples from Region 2 differed distinctly from parameters measured for samples from the other regions. The obtained values confirmed good reservoir properties of the sandstones from Region 2, as the area of the tight gas Trzek well prospecting success.

Computed microtomography and NMR results obtained in this study enabled us to gather information about the size and tortuosity of pore channels, to be combined with free water and capillary water saturation. Pore structure homogeneity was also presented as important factor.

Processing of 3D images, which were obtained with computed X-ray microtomography, gave many parameters, later used to parametrize the pore space. Some parameters (e.g. porosity, mean chord length), obtained from the micro-CT method, correlated well with effective porosity, obtained from NMR. Therefore, the prediction of porosity and permeability, on the basis of the results of the micro-CT method and the description of the pore space obtained from the computed microtomography, was more complete than that given by porosimetry.

In future, it will be essential to be particular about the homogeneity of pores distribution, during laboratory measurements. This was of great importance in the evaluation of the repre- sentativeness of data from examined small rock fragments, used in the computed X-ray microtomography and mercury injection porosimetry. Caution was exercised when predicting reservoir properties of samples with heterogeneous pore structure. On the account of quite different physical phenomena that were employed by different measurement facilities, it was important to apply as many research methods as possible. This provided complementary information on the pore space of examined rocks.

Computed X-ray microtomography gave differentiation of the investigated areas, with respect to their pore structure. The results enabled us to prepare a sophisticated discussion about the various factors related to porosity development. Micro-CT images and calculated parameters provide new information about rocks, which is very detailed, due to micro-scale investigation. This information and data cannot be obtained by other methods. Despite the relatively high costs of the investigations, the micro-CT method should be proposed as a standard measurement in reservoir properties determination. It is an important support instrument in specialist investigations.

This paper was written based on the research made by Marek Dohnalik to prepare his Ph.D. thesis. The Benchtop CT160Xi device in Oil and Gas Institute, Cracow, and software ImageJ, AVIZO, and MAVI were used.

\section{REFERENCES}

Akkurt R., Vinegar H.J., Tutunjian P.N. \& Guillory A.J., 1995. NMR logging of natural gas reservoirs. Transactions of the SPWLA 36th Annual Logging Symposium, 26-29 June 1995, Paris, France, paper N, Society of Professional Well Log Analysts.

Aksamitowska A., 2003. Środowiska sedymentacji i rozwój depozycji osadów górnego czerwonego spągowca w rejonie Poznania. Przegląd Geologiczny, 51, 2, 168-174.

Buniak A. \& Mikołajewski Z., 1997. Środowiska depozycyjne, petrografia i diageneza osadów czerwonego spągowca w rejonie Poznania. Geologos, 2, 201-214.

Buniak A., Kiersnowski H. \& Kuberska M., 2008a. Perspektywy poszukiwań złóż gazu ziemnego w piaskowcach czerwonego spągowca o słabych właściwościach zbiornikowych w strefie Poznań-Konin-Kalisz. IV Krajowy Zjazd Branży Górnictwa Naftowego, Materiały konferencyjne, Łagów Lubuski, 11-13.09.2008, 125-138.

Buniak A., Kwolek K., Kiersnowski H. \& Kuberska M., 2008b. Perspektywy odkrycia złóż gazu ziemnego (typu tight gas) 
w piaskowcach eolicznych w basenie górnego czerwonego spągowca. Międzynarodowa Konferencja Naukowo-Techniczna Geopetrol 2008 nt. Nauka, technika i technologia $w$ rozwoju poszukiwań $i$ wydobycia weglowodorów $w$ warunkach lądowych i morskich. Zakopane, 15-18.09.2008, series: Prace Instytutu Nafty i Gazu, 150, Instytut Nafty i Gazu, Kraków, 61-66.

Buniak A., Kuberska M. \& Kiersnowski H., 2009. Petrograficzno-petrofizyczna charakterystyka piaskowców eolicznych strefy Siekierki-Winna Góra (koło Poznania) w aspekcie poszukiwań złóż gazu zamkniętego w osadach czerwonego spągowca. Przegląd Geologiczny, 57, 4, 328-334.

Cnudde V., 2005. Exploring the potential of X-ray tomography as a new non-destructive research tool in conservation studies of natural building stones. IWT-Vlaanderen, Belgium.

Coates G.R., Lizhi X. \& Prammer M.G., 1999. NMR logging. Principles and Interpretation. Halliburton Energy Service, Huston, Texas.

Dohnalik M. \& Zalewska J., 2008. Zastosowanie mikrotomografii rentgenowskiej do rozwiązywania zagadnień geologicznych i geofizycznych. Instytut Nafty i Gazu - Państwowy Instytut Badawczy, Kraków, nr DK-4100-15/08 [research and development work].

Dohnalik M., Zalewska J. \& Kaczmarczyk J., 2009. Ilościowa analiza parametrów wewnętrznej struktury porowej skał metoda rentgenowskiej mikrotomografii komputerowej. Instytut Nafty i Gazu - Państwowy Instytut Badawczy, Kraków [research and development work].

Dong H., Fjeldstad S., Alberts L., Roth S., Bakke S. \& Øren P.-E., 2008. Pore network modelling on carbonate: a comparative study of different micro-CT Network extraction methods. International Symposium of the Society of Core Analysts Abu Dhabi, UAE 29 October-2 November, 2008.

Fisher R., Perkins S., Walker A. and Wolfart E., 2003: A to $Z$ of Image Processing Concepts, pixel connectivity. [on-line:] http://homepages.inf.ed.ac.uk/rbf/HIPR2/glossary.htm [access: September 2011].

Golab A., Ward Colin R., Permana A., Lennox P. \& Botha P., 2013. High-resolution three-dimensional imaging of coal using microfocus X-ray computed tomography, with special reference to modes of mineral occurrence. International Journal of Coal Geology, 113, 97-108.

Karnkowski P.H., 1999. Wprowadzenie do konferencji pt. Polski basen czerwonego spągowca - geologia i złoża gazu ziemnego. Przegląd Geologiczny, 47, 5, 461-470.

Kiersnowski H., 1997. Depositional development of the Polish Upper Rotliegend Basin and evolution of its sediment source areas. Geological Quarterly, 41, 4, 433-456.

Kiersnowski H., 1998. Architektura depozycyjna basenu czerwonego spągowca w Polsce, Analiza basenów sedymentacyjnych Niżu Polskiego. [in:] Narkiewicz M. (red.), Analiza basenów sedymentacyjnych Niżu Polskiego, series: Prace Państwowego Instytutu Geologicznego, 165, PIG, Warszawa, 113-128.

Kiersnowski H., Buniak A., Kuberska M. \& Srokowska-Okońska A., 2010. Występowanie gazu ziemnego zamkniętego w piaskowcach czerwonego spągowca Polski. Przegląd Geologiczny, 58, 4, 335-346.

Klaja J. \& Gąsior I., 2010. Ilościowa ocena przestrzeni porowej zajętej wodą związaną w iłach, z wykorzystaniem metody magnetycznego rezonansu jądrowego. Nafta Gaz, 3, 178-188.

Kwolek K., Solarski T. \& Buniak A., 2004. Poszukiwanie pulapek litologicznych w utworach czerwonego spągowca na NE skłonie wału wolsztyńskiego. Nafta Gaz, 9, 406-415.

Law B.E. \& Spencer C.W., 1993. Gas in tight reservoirs-an emerging major source of energy. [in:] Howell D.G. (ed.), The Future of Energy Gasses, US Geological Survey, Professional Paper 1570, 233-252.

Micromeritics.com, [on-line:] http://www.micromeritics. com/Product-Showcase/AccuPyc-II-1340.aspx [access: September 2012].

Młynarczuk M., 2004: Zastosowanie automatycznej analizy obrazów w pomiarach struktur skał. Geotechnika i budownictwo specjalne 2004: XXVII Zimowa Szkoła Mechaniki Górotworu, Zakopane 14-19 marca 2004, KGBiG AGH, Kraków, 305-318, [on-line:] http://www.teberia. $\mathrm{pl} /$ bibliografia.php? $\mathrm{a}=$ showarticle \&ArticleID $=11162$ [access: May 2010].

Mutina A. \& Bruyndonckx P., 2013. Combined micro-X-ray tomography and micro-X-ray fluorescence study of reservoir rocks: applicability to core analysis. Microscopy and Analysis - Compositional Analysis Supplement, 27, 4, S4-S6.

Nadeev A., Mikhailov D., Chuvilin E., Koroteev D. \& Shako V., 2013. Visualization of clay and frozen substances inside porous rocks using X-ray micro-computed tomography. Microscopy and Analysis - Tomography Supplement, 27, 2, S8-S11.

Osher J. \& Schladitz K., 2009. 3D Images of Material Structures: Processing and Analysis. Wiley-Vch Verlag GmbH \& Co. KGaA.

Peitgen H.-O., Äurgens H.J. \& Saupe D., 1995: Fraktale: granice chaosu. Cz. 1. Wydawnictwo Naukowe PWN, Warszawa.

Pokorski J., 1997. Perm dolny (czerwony spagowiec). [in:] Marek S. \& Pajchlowa M., Epikontynentalny perm i mezozoik $w$ Polsce, series: Prace Państwowego Instytutu Geologicznego, 153, PIG, Warszawa, 35-62.

Raport z otworów: Czarna Wieś-6, 7, Parzęczewo-1, 2, Parzęczewo-1, Środa Wielkopolska-6, Kromolice-1, 2, Siekierki 3,4, Miłosław 2. Archiwum PGNiG S.A., Warszawa, Polska.

San Leon Energy, [on-line]: http://www.sanleonenergy.com/ search.aspx?search_string $=$ siekierki\& $\mathrm{x}=0 \& \mathrm{y}=0$ [access: July 2014].

Sato M., Bitter I., Bender M., Kaufnab A. \& Nakajima M., 2000. TEASAR: Tree Structure Extraction Algorithm for Accurate and Robust Skeletons. The Eighth Pacific Conference on Computer Graphics and Applications, 2000. Proceedings, Hong Kong, 281-449.

Straley C., Rossini D., Vinegar H., Tutunjian P. \& Morriss C., 1997. Core analysis by low field NMR. The Log Analyst, March-April, 84-94.

Toriwaki J. \& Yonekura T., 2002. Euler Number and Connectivity Indexes of a Three Dimensional Digital Picture. Forma, 17, 183-209.

Tsakiroglu Ch.D. \& Payatakes A.C., 2000. Characterization of the pore structure of reservoir rocks with the aid of serial sectioning analysis, mercury porosimetry and network simulation. Advances in Water Resources, 23, 773-789. 
Vogel H.-J., 2002. Morphology of Condensed Matter, Physics and Geometry of Spatially Complex Systems. [in:] Mecke K. \& Stoyan D. (eds), Topological Characterization of Porous Media, Springer-Verlag, Berlin, Heidelberg, 75-92.

Xiao L., Mao Z. Q., Xiao Z. X. \& Zhang C., 2008. A new method to evaluate reservoir pore structure consecutively using NMR and capillary pressure data. SPWLA 49th Annual Logging Symposium, May 25-28, 2008, 1-6

Yang X., 2005. Three-Dimensional characterization of inherent and induced sand microstructure. Georgia Institute of Technology, USA [Ph.D. thesis].
Zalewska J., Dohnalik M., Cebulski D., Kowalska S., Klaja J. \& Łykowska G., 2009. Trójwymiarowa wizualizacja struktury przestrzeni porowej skat metoda mikrotomografii komputerowej (micro-CT) piaskowców czerwonego spagowca z rejonu Cicha Góra Czarna Wieś - Parzęczewo. Archiwum Instytutu Nafty i Gazu, Kraków, nr INiG DK-4100-145/08 [research work].

Ziemianin K., 2012. Archiwum Instytutu Nafty i Gazu, Kraków [unpublished research results performed in the Department of Geology and Geochemistry of Oil and Gas Institute]. 